\title{
Abelian decomposition of Einstein's theory: Reformulation of general relativity
}

\author{
Y. M. Cho $*$ Sang-Woo Kim $才$ and J. H. Kim \\ Center for Theoretical Physics and School of Physics, \\ College of Natural Sciences, \\ Seoul National University, Seoul 151-742, Korea
}

\begin{abstract}
We propose a reformulation of general relativity by making the Abelian decomposition of Einstein's theory. Based on the view that Einstein's theory can be interpreted as a gauge theory of Lorentz group, we decompose the gravitational connection (the gauge potential of Lorentz group $\boldsymbol{\Gamma}_{\mu}$ ) into the restricted connection made of the potential of the maximal Abelian subgroup $H$ of Lorentz group $G$ and the valence connection made of $G / H$ part of the potential which transforms covariantly under Lorentz group. With this decomposition we show that the Einstein's theory can be decomposed into the restricted part made of the restricted connection which has the full Lorentz gauge invariance and the valence part made of the valence connection which plays the role of gravitational source of the restricted gravity. We show that there are two different Abelian decomposition of Einstein's theory, the space-like decomposition and the light-like decomposition, because Lorentz group has two maximal Abelian subgroups. In this decomposition the role of the space-time metric $g_{\mu \nu}$ is replaced by a Lorentz covariant four-index metric tensor $\mathbf{g}_{\mu \nu}$ which transforms covariantly under the Lorentz group, and the metric-compatibility condition $\nabla_{\alpha} g_{\mu \nu}=0$ of the connection is replaced by the gauge covariant condition $D_{\mu} \mathbf{g}^{\mu \nu}=0$ which tells that $\mathbf{g}_{\mu \nu}$ is invariant under the parallel transport along the $\partial_{\mu}$-direction defined by $\boldsymbol{\Gamma}_{\mu}$. We discuss the physical implications of the Abelian decomposition. In particular, we argue that the decomposition implies the existence of a restricted theory of gravitation which has the full general invariance but has less physical degrees of freedom.

PACS numbers:
\end{abstract}

Keywords: Abelian decomposition of Einstein's theory, restricted gravity

\section{INTRODUCTION}

Einstein's theory of gravitation and the gauge theory (Abelian and non-Abelian) are two fundamental ingredients of theoretical physics which have played a crucial role to advance our understanding of nature. Einstein's theory has been very successful describing the gravitational force. At the same time it has played a crucial role in unified theory [1, 2]. In fact all modern unified theories, including the superstring, are based on Einstein's theory one way or another. This originates from the general invariance principle of Einstein's theory which guarantees that all physical laws should be independent of the observers. For this reason Einstein's theory has been the subject of intensive theoretical study.

The gauge theory, the other important ingredient in theoretical physics, describes the electroweak and strong forces. But the gauge theory is closely related to Einstein's theory. Indeed the gauge theory itself can be viewed as an Einstein's theory originating from the extrinsic curvature of $(4+n)$-dimensional uni-

\footnotetext{
*Electronic address: ymcho@yongmin.snu.ac.kr

${ }^{\dagger}$ Electronic address: eyed@phya.snu.ac.kr

‡Electronic address: rtiger@phya.snu.ac.kr
}

fied space. It is well-known that the $(4+n)$-dimensional unified space made of the 4-dimensional space-time and an n-dimensional internal space, the $(4+n)$-dimensional Einstein's theory reproduces the gauge theory when the internal space has an n-dimensional isometry $G$. In fact the $(4+n)$-dimensional Einstein's theory provides a natural unification of gauge theory with gravitation, which is known as the Kaluza-Klein miracle [1, 2, 3, 4, 5].

Conversely Einstein's theory itself can be understood as a gauge theory, because the general invariance of Einstein's theory can be viewed as a gauge invariance. The idea that the general invariance can be associated to a gauge invariance has been proposed by many people, but the actual application of this idea to Einstein's theory has been non-trivial [6, 7, 8, 9, 10, 11]. There are two ways to formulate the Einstein's theory as a gauge theory. One can view it as a gauge theory of 4-dimensional translation group, because the local 4-dimensional translation can be identified as the general coordinate transformation [6]. In this case one can identify the gauge potential of the translation group as the (non-trivial part of) the tetrad [9]. Or, one can view it as a gauge theory of Lorentz group (or Poincare group in general), because the Lorentz gauge transformation can also be interpreted as the general coordinate transformation [7, 8]. In this case one can identify the gauge potential of Lorentz group as the spin connection [10, 11]. This tells that the two theories are closely related. 
During the last few decades our understanding of nonAbelian gauge theory has been extended very much. By now it has been well known that the non-Abelian gauge potential allows the Abelian decomposition [12, 13]. It can be decomposed into the restricted potential of the maximal Abelian subgroup $H$ of the gauge group $G$ which has an electric-magnetic duality and the valence potential of $G / H$ which transforms covariantly under $G$. A remarkable feature of this decomposition is that it is gauge independent. As importantly, the restricted potential has the full non-Abelian gauge degrees of freedom, in particular the topological degrees of the gauge group $G$, in spite of the fact that it consists of only the Abelian degrees of the maximal Abelian subgroup $H$. This means that we can construct a restricted gauge theory, a non-Abelian gauge theory made of only the restricted potential which has much less physical degrees of freedom, which nevertheless has the full gauge invariance. Furthermore, we can recover the full non-Abelian gauge theory simply by adding the valence part. This tells that the non-Abelian gauge theory can be interpreted as a restricted gauge theory which has the valence potential as the gauge covariant source [12, 13]. The importance of this decomposition is that the restricted part plays a fundamental role for us to establish the Abelian dominance in nonAbelian dynamics, which have been pivotal in proving the monopole condensation and confinement of color in QCD [14, 15, 16, 17].

The purpose of this paper is to present a similar Abelian decomposition of Einstein's theory, regarding the theory as a gauge theory of Lorentz group. Applying the Abelian decomposition to the gauge potential of Lorentz group, we show that we can decompose the gravitational connection to the restricted connection and the valence connection. With this we decompose the Einstein's theory into the restricted part made of the restricted connection and the valence part made of the gauge covariant valence connection. The decomposition tells that the Einstein's theory can be viewed as a restricted theory of gravitation which has the gauge covariant valence connection as the gravitational source. We show that Einstein's theory allows two different Abelian decompositions, the space-like decomposition and the light-like decomposition, because the Lorentz group has two maximal Abelian subgroups. To decompose the Einstein's theory, we introduce the concept of the gauge covariant metric $\mathbf{g}_{\mu \nu}$, a four-index tensor $g_{\mu \nu}{ }^{a b}$ which forms an adjoint representation of Lorentz group, and show that the metric-compatibility condition of the gravitational connection $\nabla_{\alpha} g_{\mu \nu}=0$ is transformed to the gauge covariant condition $D_{\mu} \mathbf{g}^{\mu \nu}=0$ which assures the invariance of $\mathbf{g}_{\mu \nu}$ under the parallel transport along the $\partial_{\mu}$-direction.

Of course, Einstein's theory as a gauge theory of Lorentz group is different from the ordinary non-Abelian gauge theory [4]. In gauge theory the fundamental field is the gauge potential, but in Einstein's theory the fundamental field is the metric. And in the gauge formu- lation the gauge potential of Lorentz group corresponds to the gravitational connection, not the metric. Also, in gauge theory the Yang-Mills Lagrangian is quadratic in field strength. But in gravitation the Einstein-Hilbert Lagrangian is made of the scalar curvature, which is linear in field strength [10]. Nevertheless we can still make the Abelian decomposition of the gravitational connection, and express the Einstein-Hilbert Lagrangian in terms of the restricted connection and the valence connection. With this we can separate the restricted part of gravitation from the Einstein's theory, and show that the theory can be interpreted as a restricted theory of gravity which has the valence connection as the gravitational source.

The paper is organized as follows. In Section II we review the geometry of gauge theory and Abelian decomposition for later purpose. In Section III we discuss the simplest Abelian decomposition, the $U(1)$ decomposition of $S U(2)$ gauge theory, as an example to help us to understand the Abelian decomposition of Einstein's theory. In Section IV we show how to decompose the gravitational connection to the Abelian part and the valence part. We show that there are two different ways of Abelian decomposition, because the Lorentz group has two maximal Abelian subgroups. In Section V introduce the concept of the Lorentz covariant metric tensor, and show how to decompose the Einstein's theory to the restricted part and the valence part. We discuss two different decompositions of Einstein's theory separately. Finally in Section VI we discuss the physical implications of our results.

\section{GEOMETRY OF GAUGE THEORY: A REVIEW}

To understand how to make the desired Abelian decomposition of Einstein's theory, we have to understand the Abelian decomposition of gauge theory first. So in this section we review the geometry of gauge theory and Abelian decomposition. Let $P$ be a $(4+\mathrm{n})$-dimensional unified space endowed with the metric $g_{A B}(A, B=$ $1,2, \ldots 4+n)$, which has an n-dimensional linearly independent isometry (the left isometry) which forms an isometry group $G$. Let the isometry be described by $n$ linearly independent vector fields $\xi_{i}$,

$$
\begin{gathered}
{\left[\xi_{i}, \xi_{j}\right]=\frac{1}{\kappa} f_{i j}{ }^{k} \xi_{k}, \quad(i, j, k=1,2, \ldots, n)} \\
\mathcal{L}_{\xi_{i}} g_{A B}=0, \quad(A, B=1,2, \ldots 4+n)
\end{gathered}
$$

where $\kappa$ is the scale parameter which determines the length of the isometry vector fields, and $\mathcal{L}_{\xi_{i}}$ is the Lie derivative along $\xi_{i}$. Clearly the integral manifold of the isometry vector fields forms an n-dimensional group manifold $G$ which acts vertically on $P$. Let $\Pi$ be the horizontal projection of $p \in P$ to $x \in M=P / G$, and identify the 4-dimensional quotient space $M$ as the space-time 
manifold. With this we can view the unified space as a $(4+\mathrm{n})$-dimensional fiber bundle $P(M, G)$ made of the 4dimensional space-time $M$ as the base manifold and the n-dimensional group space $G$ as the fiber space on which $G$ acts as the structural group [3, 4] .

Let $\partial_{\mu}(\mu=0,1,2,3)$ be a coordinate basis on $M$,

$$
\left[\partial_{\mu}, \partial_{\nu}\right]=0
$$

and $D_{\mu}=\Pi^{-1} \cdot \partial_{\mu}$ be the horizontal lift of $\partial_{\mu}$ on $P$. Clearly $D_{\mu}$ and $\xi_{i}$ forms a basis of $P$ which has the following commutation relations [3, 4],

$$
\begin{gathered}
{\left[D_{\mu}, D_{\nu}\right]=-\kappa F_{\mu \nu}{ }^{k} \xi_{k},} \\
{\left[D_{\mu}, \xi_{i}\right]=0, \quad\left[\xi_{i}, \xi_{j}\right]=\frac{1}{\kappa} f_{i j}{ }^{k} \xi_{k} .}
\end{gathered}
$$

Moreover, since $D_{\mu}$ are horizontal, we have

$$
\begin{gathered}
g_{A B} D_{\mu}^{A} D_{\nu}^{B}=\gamma_{\mu \nu}, \\
g_{A B} D_{\mu}{ }^{A} \xi_{i}{ }^{B}=0 \\
g_{A B} \xi_{i}{ }^{A} \xi_{j}{ }^{B}=\phi_{i j} .
\end{gathered}
$$

So, in the basis (3) the metric acquires the following form

$$
g_{A B}=\left(\begin{array}{cc}
\gamma_{\mu \nu} & 0 \\
0 & \phi_{i j}
\end{array}\right)
$$

This suggests us to identify $g_{\mu \nu}$ and $\phi_{i j}$ as the 4dimensional metric on the space-time manifold $M$ and the n-dimensional internal metric on the fiber space $G$. From (3) and (5) we can calculate the Einstein-Hilbert action on $P$. With

$$
\begin{gathered}
g=\operatorname{Det}\left(g_{\mu \nu}\right), \quad \phi=\operatorname{Det}\left(\phi_{i j}\right), \\
\phi_{i j}=\phi^{1 / n} \rho_{i j}, \quad\left|\operatorname{Det} \rho_{i j}\right|=1,
\end{gathered}
$$

we have (up to a total divergence) [3, 4]

$$
\begin{gathered}
S_{P}=-\frac{V_{G}}{16 \pi G_{P}} \int \sqrt{-} \gamma \sqrt{\phi}\left\{R_{M}+R_{G}\right. \\
-\frac{\kappa^{2}}{4} \phi^{1 / n} \rho_{i j} \gamma^{\mu \rho} \gamma^{\nu \sigma} F_{\mu \nu}{ }^{i} F_{\rho \sigma}^{j} \\
+\frac{1}{4} \rho^{i j} \rho^{k l} \gamma^{\mu \nu}\left(D_{\mu} \rho_{i k}\right)\left(D_{\nu} \rho_{j l}\right)-\frac{n-1}{4 n} \frac{\gamma^{\mu \nu}\left(\partial_{\mu} \phi\right)\left(\partial_{\nu} \phi\right)}{\phi^{2}} \\
\left.-\Lambda_{P}+\lambda\left(\left|\operatorname{Det} \rho_{i j}\right|-1\right)\right\} d^{4} x
\end{gathered}
$$

where $G_{P}$ and $\Lambda_{P}$ are the $(4+\mathrm{n})$-dimensional gravitational constant and cosmological constant of $P, V_{G}$ is the n-dimensional volume of the group space $G, R_{M}$ and $R_{G}$ are the scalar curvatures of the 4-dimensional metric $\gamma_{\mu \nu}$ and the n-dimensional metric $\phi_{i j}$.

To clarify the meaning of the unified action (14) we introduce another basis defined by a cross section $\sigma(x)$ in $P$. Let $U$ be an open subset of $M$, and $\sigma(x)$ be a 4dimensional submanifold in $P$ which is diffeomorphic to
$U$ with $\Pi \cdot \sigma(x)=x \in U$. Clearly we can view $\sigma$ as a mapping from $U$ to $\sigma \subset P$,

$$
\sigma: \quad x \in U \rightarrow \sigma(x) \in \Pi^{-1}(U) .
$$

Let $\tilde{\partial}_{\mu}{ }^{(\sigma)}=\sigma \cdot \partial_{\mu}$ be the mapping of the vector field $\partial_{\mu}$ on $\sigma(x)$ induced by $\Pi^{-1}$, and let $\tilde{\partial}_{\mu}$ be the vector field induced on $\Pi^{-1}(U)$ obtained by the vertical translation of $\tilde{\partial}_{\mu}{ }^{(\sigma)}$. Clearly $\tilde{\partial}_{\mu}$ and $\xi_{i}$ form a basis on $\Pi^{-1}(U)$ which has the following commutation relations

$$
\begin{gathered}
{\left[\tilde{\partial}_{\mu}, \tilde{\partial}_{\nu}\right]=0,} \\
{\left[\tilde{\partial}_{\mu}, \xi_{i}\right]=0, \quad\left[\xi_{i}, \xi_{j}\right]=\frac{1}{\kappa} f_{i j}{ }^{k} \xi_{k} .}
\end{gathered}
$$

Let

$$
\begin{gathered}
D_{\mu}=\tilde{\partial}_{\mu}-\kappa A_{\mu}{ }^{i} \xi_{i}, \\
g_{A B} \xi_{i}{ }^{A} \tilde{\partial}_{\mu}{ }^{B}=\kappa \phi_{i j} A_{\mu}{ }^{j},
\end{gathered}
$$

and find that in this basis the metric is written as

$$
g_{A B}=\left(\begin{array}{cc}
g_{\mu \nu} & \kappa A_{\mu}^{i} \phi_{i j} \\
\kappa \phi_{i j} A_{\nu}^{j} & \phi_{i j}
\end{array}\right) \text {. }
$$

Moreover, with (9) and (10) we have

$$
\begin{gathered}
\partial_{\xi_{i}} A_{\mu}{ }^{k}=-\frac{1}{\kappa} f_{i j}{ }^{k} A_{\mu}{ }^{j}, \\
F_{\mu \nu}{ }^{k}=\partial_{\mu} A_{\nu}{ }^{k}-\partial_{\nu} A_{\mu}{ }^{k}+f_{i j}{ }^{k} A_{\mu}{ }^{i} A_{\nu}{ }^{j} .
\end{gathered}
$$

This tells that $A_{\mu}^{k}$ and $F_{\mu \nu}{ }^{k}$ can be interpreted as the gauge potential and the field strength of the isometry group $G$ (in which the coupling constant $g$ is normalized to be the unit), and the horizontal lift basis $D_{\mu}$ plays the role of the gauge covariant derivative.

Now, with

$$
g_{\mu \nu}=\sqrt{\phi} \gamma_{\mu \nu}, \quad \varphi=\frac{1}{2} \sqrt{\frac{n+2}{n}} \ln \phi,
$$

(14) is written as

$$
\begin{gathered}
S_{P}=-\frac{\sqrt{-} g}{16 \pi G_{N}} \int\left\{R_{4}+\frac{1}{2} g^{\mu \nu}\left(\partial_{\mu} \varphi\right)\left(\partial_{\nu} \varphi\right)\right. \\
+\hat{R}_{G} \exp \left(-\frac{n+2}{n} \varphi\right)-\Lambda_{P} \exp \left(-\frac{n}{n+2} \varphi\right) \\
-\frac{\kappa^{2}}{4} \exp \left(\frac{n+2}{n} \varphi\right) \rho_{i j} g^{\mu \rho} g^{\nu \sigma} F_{\mu \nu}{ }^{i} F_{\rho \sigma}^{j} \\
+\frac{1}{4} \rho^{i j} \rho^{k l} g^{\mu \nu}\left(D_{\mu} \rho_{i k}\right)\left(D_{\nu} \rho_{j l}\right) \\
\left.+\lambda\left(\left|\operatorname{Det} \rho_{i j}\right|-1\right)\right\} d^{4} x,
\end{gathered}
$$

where $\hat{R}_{G}$ is the scalar curvature of the normalised metric $\rho_{a b}$

$$
\hat{R}_{G}\left(\rho_{a b}\right)=-\frac{1}{2} f_{a b}^{d} f_{c d}^{b} \rho^{a c}-\frac{1}{4} f_{a b}^{m} f_{c d}^{n} \rho^{a c} \rho^{b d} \rho_{m n}
$$


This tells that the $(4+\mathrm{n})$-dimensional Einstein's theory indeed describes the unified theory of the 4-dimensional Einstein's theory and the gauge theory of the isometry group $G$ coupled to the gauge covariant internal metric $\phi_{i j}$, provided that $[3,4]$

$$
\frac{V_{G}}{16 \pi G_{P}}=\frac{1}{16 \pi G_{N}}, \quad \frac{\kappa^{2}}{16 \pi G_{N}}=1
$$

where $G_{N}$ is the 4-dimensional Newton's gravitational constant. In this unification the isometry group $G$ plays the role of the gauge group, and the choice of a cross section corresponds to the choice of a gauge 3, 4]. Notice, however, that the metric $g_{\mu \nu}$ on $M$ is the Jordan metric, not the Pauli metric which describes the spin-2 graviton. To obtain the metric which describes the spin- 2 graviton one need to make a proper conformal transformation of the Jordan metric [18]. $\delta_{i j}$

Notice that, when $\phi_{i j}$ becomes the bi-invariant metric

$$
\phi_{i j}=\delta_{i j}=-\frac{1}{2} f_{i k}^{l} f_{j l}^{k}
$$

the scalar source $\phi_{i j}$ disappears completely from (14), and the $(4+n)$-dimensional Einstein-Hilbert action is simplified to

$$
\begin{gathered}
S_{P}=-\frac{1}{16 \pi G_{N}} \int \sqrt{-} g\left(R_{M}-\frac{1}{4} \vec{F}_{\mu \nu}^{2}\right. \\
\left.+R_{G}-\Lambda_{P}\right) d^{4} x,
\end{gathered}
$$

where $R_{M}$ and $R_{G}$ are the scalar curvature of the 4dimensional space-time and the n-dimensional internal space. And $R_{G}$ (with $\phi_{i j}=\delta_{i j}$ ) becomes a constant. This provides the $(4+\mathrm{n})$-dimensional unification of gravitation and gauge theory with no other source, where $R_{G}-\Lambda_{P}=\Lambda_{M}$ now plays the role of the 4-dimensional cosmological constant [3, 4$]$.

We emphasize that in this unification the volume of the fiber space is space-time dependent, because $\phi=$ $\phi(x)$. More importantly, the volume of the group space $V_{G}$ (relative to the volume of the space-time $V_{M}$ ) is left arbitrary, so that the internal space can have any size. In spite of this the internal space becomes completely unphysical and unobservable. This is because this unification is the unification by isometry, in which the internal space becomes unobservable because of the exact isometry [3, 4, 5]. And there is no "higher modes" of internal metric in this unification. In comparison, in the popular higher-dimensional unification where the internal space has no isometry, the internal space becomes "unobservable" only when it is small (of the order of the Planck scale) [19]. Moreover, one has to deal with the "higher modes" of internal metric in the popular unification. We believe that our unification by isometry provides the only logically consistent higher-dimensional unification of gauge theory with gravitation [20].
With this preliminary, we now discuss the geometry of Abelian decomposition. Clearly any extra isometry on $P$ will further restrict the metric $g_{A B}$, and thus reduce the physical degrees of the unified theory. Suppose we have an extra right isometry (the magnetic isometry) $H$ which is vertical, which commutes with the left isometry $G$. Let the right isometry be described by $m_{a}$,

$$
\begin{gathered}
\forall_{a} \quad \mathcal{L}_{m_{a}} g_{A B}=0, \\
m_{a}=m_{a}{ }^{i} \xi_{i}, \\
{\left[m_{a}, m_{b}\right]=\bar{f}_{a b}{ }^{c} m_{c},} \\
{\left[m_{a}, \xi_{i}\right]=0 . \quad(a, b, c=1,2, \ldots, m<n)}
\end{gathered}
$$

Clearly the last commutation relation tells that $\hat{m}_{a}=$ $\left(m_{a}{ }^{1}, m_{a}{ }^{2}, \ldots, m_{a}{ }^{n}\right)$ forms an adjoint representation of $G$. Furthermore, when $\phi_{i j}=\delta_{i j}$, the magnetic isometry can be written as 12 .

$$
\begin{aligned}
& \forall_{a} \quad D_{\mu} \hat{m}_{a}=0, \\
& \hat{m}_{a} \times \hat{m}_{b}=\hat{m}_{c} .
\end{aligned}
$$

This condition restricts the gauge potential, and defines the restricted potential which satisfies the extra magnetic isometry.

Let $H$ be the maximal Abelian subgroup of $G$, and let $\hat{A}_{\mu}$ be the restricted potential which satisfies the above condition. Then the most general gauge potential $\vec{A}_{\mu}$ can be written as

$$
\vec{A}_{\mu}=\hat{A}_{\mu}+\vec{X}_{\mu}
$$

where $\vec{X}_{\mu}$ is the $G / H$ part of potential which transforms covariantly under the gauge transformation. This is because the space of gauge potentials (the connection space) forms an Affine space, so that one can obtain arbitrary potential by adding a gauge covariant vector field to the restricted potential. This allows us to identify $\vec{X}_{\mu}$ as the valence potential. This decomposition of gauge potential is called the Abelian decomposition [12, 15].

The above Abelian decomposition has important features. First the restricted potential $\hat{A}_{\mu}$ enjoys the full gauge symmetry of $G$, in spite of the fact that it is restricted. This is because the left isometry $G$ (the full gauge symmetry) remains intact in this decomposition [3, 4, 5]. Secondly, it retains all essential topological properties of $G$. In particular, $\hat{A}_{\mu}$ naturally selects the magnetic potential which describes the non-Abelian monopole. For this reason the right isometry (19) is called the magnetic isometry. But most importantly, the decomposition is gauge independent. Once $H$ is chosen, the decomposition follows automatically, independent of the choice of a gauge. 


\section{ABELIAN DECOMPOSITION OF $S U(2)$ : AN EXAMPLE}

To show how the Abelian decomposition works, we consider the $S U(2)$ gauge theory for simplicity. Let $\hat{n}$ be an arbitrary isotriplet unit vector field, and identify the maximal Abelian subgroup to be the $U(1)$ subgroup which leaves $\hat{n}$ invariant. Clearly $\hat{n}$ selects the "Abelian" direction (i.e., the color charge direction) at each spacetime point, and the the Abelian magnetic isometry can be described by the following constraint equation

$$
D_{\mu} \hat{n}=\partial_{\mu} \hat{n}+g \vec{A}_{\mu} \times \hat{n}=0 . \quad\left(\hat{n}^{2}=1\right)
$$

This has the unique solution for $\vec{A}_{\mu}$ which defines the restricted potential $\hat{A}_{\mu}$

$$
\hat{A}_{\mu}=A_{\mu} \hat{n}-\frac{1}{g} \hat{n} \times \partial_{\mu} \hat{n},
$$

where $A_{\mu}=\hat{n} \cdot \vec{A}_{\mu}$ is the "electric" potential. Notice that the restricted potential is precisely the connection which leaves $\hat{n}$ invariant under the parallel transport,

$$
\hat{D}_{\mu} \hat{n}=\partial_{\mu} \hat{n}+g \hat{A}_{\mu} \times \hat{n}=0 .
$$

This process of selecting the restricted potential is called the Abelian projection [12, 13].

With the Abelian projection we can retrieve the full gauge potential by adding the gauge covariant valence potential $\vec{X}_{\mu}$ to the restricted potential,

$$
\begin{gathered}
\vec{A}_{\mu}=A_{\mu} \hat{n}-\frac{1}{g} \hat{n} \times \partial_{\mu} \hat{n}+\vec{X}_{\mu}=\hat{A}_{\mu}+\vec{X}_{\mu} \\
\left(\hat{n}^{2}=1, \quad \hat{n} \cdot \vec{X}_{\mu}=0\right) .
\end{gathered}
$$

This is the Abelian decomposition which decomposes the gauge potential into the restricted potential $\hat{A}_{\mu}$ and the valence potential $\vec{X}_{\mu}[12,13]$.

Let $\vec{\alpha}$ is an infinitesimal gauge parameter. Under the infinitesimal gauge transformation

$$
\delta \hat{n}=-\vec{\alpha} \times \hat{n}, \quad \delta \vec{A}_{\mu}=\frac{1}{g} D_{\mu} \vec{\alpha}
$$

one has

$$
\begin{gathered}
\delta A_{\mu}=\frac{1}{g} \hat{n} \cdot \partial_{\mu} \vec{\alpha}, \quad \delta \hat{A}_{\mu}=\frac{1}{g} \hat{D}_{\mu} \vec{\alpha} \\
\delta \vec{X}_{\mu}=-\vec{\alpha} \times \vec{X}_{\mu} .
\end{gathered}
$$

This shows that $\hat{A}_{\mu}$ by itself describes an $S U(2)$ connection which enjoys the full $S U(2)$ gauge degrees of freedom. Furthermore $\vec{X}_{\mu}$ transforms covariantly under the gauge transformation. Most importantly, the decomposition is gauge-independent. Once the color direction $\hat{n}$ is selected, the decomposition follows independent of the choice of a gauge.
To understand the physical meaning of our decomposition notice that the restricted potential $\hat{A}_{\mu}$ actually has a dual structure. Indeed the field strength made of the restricted potential is decomposed as

$$
\begin{gathered}
\hat{F}_{\mu \nu}=\partial_{\mu} \hat{A}_{\nu}-\partial_{\nu} \hat{A}_{\mu}+g \hat{A}_{\mu} \times \hat{A}_{\nu} \\
=\left(F_{\mu \nu}+H_{\mu \nu}\right) \hat{n}, \\
F_{\mu \nu}=\partial_{\mu} A_{\nu}-\partial_{\nu} A_{\mu}, \\
H_{\mu \nu}=-\frac{1}{g} \hat{n} \cdot\left(\partial_{\mu} \hat{n} \times \partial_{\nu} \hat{n}\right)=\partial_{\mu} \widetilde{C}_{\nu}-\partial_{\nu} \widetilde{C}_{\mu},
\end{gathered}
$$

where $\widetilde{C}_{\mu}$ is the "magnetic" potential [12, 13]. Notice that we can always introduce the magnetic potential (at least locally section-wise), because $H_{\mu \nu}$ forms a closed two-form

$$
\partial_{\mu} H_{\mu \nu}^{d}=0 \quad\left(H_{\mu \nu}^{d}=\frac{1}{2} \epsilon_{\mu \nu \rho \sigma} H_{\rho \sigma}\right) .
$$

This allows us to identify the non-Abelian magnetic potential by

$$
\vec{C}_{\mu}=-\frac{1}{g} \hat{n} \times \partial_{\mu} \hat{n},
$$

in terms of which the magnetic field strength is expressed as

$$
\begin{gathered}
\vec{H}_{\mu \nu}=\partial_{\mu} \vec{C}_{\nu}-\partial_{\nu} \vec{C}_{\mu}+g \vec{C}_{\mu} \times \vec{C}_{\nu} \\
=-g \vec{C}_{\mu} \times \vec{C}_{\nu}=-\frac{1}{g} \partial_{\mu} \hat{n} \times \partial_{\nu} \hat{n}=H_{\mu \nu} \hat{n}
\end{gathered}
$$

As importantly $\hat{A}_{\mu}$, as an $S U(2)$ potential, retains all the essential topological characteristics of the original non-Abelian potential. This is because the topological field $\hat{n}$ naturally represents the non-Abelian topology $\pi_{2}\left(S^{2}\right)$ which describes the mapping from an $S^{2}$ in 3dimensional space $R^{3}$ to the coset space $S U(2) / U(1)$, and $\pi_{3}\left(S^{3}\right) \simeq \pi_{3}\left(S^{2}\right)$ which describes the mapping from the compactified 3-dimensional space $S^{3}$ to the group space $S^{3}$. Clearly the isolated singularities of $\hat{n}$ defines $\pi_{2}\left(S^{2}\right)$ which describes the non-Abelian monopoles. Indeed $\vec{C}_{\mu}$ with $\hat{n}=\hat{r}$ describes precisely the Wu-Yang monopole [12, 21]. This is why we call $\vec{C}_{\mu}$ the magnetic potential. Besides, with the $S^{3}$ compactification of $R^{3}, \hat{n}$ characterizes the Hopf invariant $\pi_{3}\left(S^{2}\right) \simeq \pi_{3}\left(S^{3}\right)$ which describes the topologically distinct vacua [22, 23, 24].

With (23) we have

$$
\vec{F}_{\mu \nu}=\hat{F}_{\mu \nu}+\hat{D}_{\mu} \vec{X}_{\nu}-\hat{D}_{\nu} \vec{X}_{\mu}+g \vec{X}_{\mu} \times \vec{X}_{\nu},
$$

so that the Yang-Mills Lagrangian is expressed as

$$
\begin{gathered}
\mathcal{L}=-\frac{1}{4} \vec{F}_{\mu \nu}^{2}=-\frac{1}{4} \hat{F}_{\mu \nu}^{2}-\frac{1}{4}\left(\hat{D}_{\mu} \vec{X}_{\nu}-\hat{D}_{\nu} \vec{X}_{\mu}\right)^{2} \\
-\frac{g}{2} \hat{F}_{\mu \nu} \cdot\left(\vec{X}_{\mu} \times \vec{X}_{\nu}\right)-\frac{g^{2}}{4}\left(\vec{X}_{\mu} \times \vec{X}_{\nu}\right)^{2} \\
+\lambda\left(\hat{n}^{2}-1\right)+\lambda_{\mu} \hat{n} \cdot \vec{X}_{\mu},
\end{gathered}
$$


where $\lambda$ and $\lambda_{\mu}$ are the Lagrangian multipliers. From the Lagrangian we have

$$
\begin{aligned}
\delta A_{\nu}: & \partial_{\mu}\left(F_{\mu \nu}+H_{\mu \nu}+X_{\mu \nu}\right) \\
& =-g \hat{n} \cdot\left\{\vec{X}_{\mu} \times\left(\hat{D}_{\mu} \vec{X}_{\nu}-\hat{D}_{\nu} \vec{X}_{\mu}\right)\right\}, \\
\delta \vec{X}_{\nu}: & \hat{D}_{\mu}\left(\hat{D}_{\mu} \vec{X}_{\nu}-\hat{D}_{\nu} \vec{X}_{\mu}\right) \\
& =g\left(F_{\mu \nu}+H_{\mu \nu}+X_{\mu \nu}\right) \hat{n} \times \vec{X}_{\mu} .
\end{aligned}
$$

where

$$
X_{\mu \nu}=g \hat{n} \cdot\left(\vec{X}_{\mu} \times \vec{X}_{\nu}\right) .
$$

Notice that here $\hat{n}$ has no equation of motion even though the Lagrangian contains it explicitly. This is because it represents a topological degrees of freedom, not a local degrees of freedom [12, 13]. From this we conclude that the non-Abelian gauge theory can be viewed as a restricted gauge theory made of the restricted potential, which has an additional colored source made of the valence gluon.

Obviously the Lagrangian (31) is invariant under the active gauge transformation (24). But notice that the decomposition introduces another gauge symmetry that we call the passive gauge transformation [15, 16],

$$
\delta \hat{n}=0, \quad \delta \vec{A}_{\mu}=\frac{1}{g} D_{\mu} \vec{\alpha},
$$

under which we have

$$
\begin{gathered}
\delta A_{\mu}=\frac{1}{g} \hat{n} \cdot D_{\mu} \vec{\alpha}, \quad \delta \hat{A}_{\mu}=\frac{1}{g}\left(\hat{n} \cdot D_{\mu} \vec{\alpha}\right) \hat{n}, \\
\delta \vec{X}_{\mu}=\frac{1}{g}\left\{D_{\mu} \vec{\alpha}-\left(\hat{n} \cdot D_{\mu} \vec{\alpha}\right) \hat{n}\right\} .
\end{gathered}
$$

This is because, for a given $\vec{A}_{\mu}$, one can have infinitely many different decomposition of (23), with different $\hat{A}_{\mu}$ and $\vec{X}_{\mu}$ choosing different $\hat{n}$. Equivalently, for a fixed $\hat{n}$, one can have infinitely many different $\vec{A}_{\mu}$ which are gauge-equivalent to each other. So our decomposition automatically induce another type of gauge invariance which comes from different choices of decomposition. This extra gauge invariance plays a crucial role in quantizing the theory [13].

An important advantage of the decomposition (23) is that it can actually "Abelianize" (or more precisely "dualize") the non-Abelian dynamics, without any gauge fixing [12, 15]. To see this let $\left(\hat{n}_{1}, \hat{n}_{2}, \hat{n}_{3}=\hat{n}\right)$ be a righthanded orthonormal basis and let

$$
\begin{gathered}
\vec{X}_{\mu}=X_{\mu}^{1} \hat{n}_{1}+X_{\mu}^{2} \hat{n}_{2}, \\
\left(X_{\mu}^{1}=\hat{n}_{1} \cdot \vec{X}_{\mu}, \quad X_{\mu}^{2}=\hat{n}_{2} \cdot \vec{X}_{\mu}\right)
\end{gathered}
$$

and find

$$
\begin{gathered}
\hat{D}_{\mu} \vec{X}_{\nu}=\left\{\partial_{\mu} X_{\nu}^{1}-g\left(A_{\mu}+\widetilde{C}_{\mu}\right) X_{\nu}^{2}\right\} \hat{n}_{1} \\
+\left\{\partial_{\mu} X_{\nu}^{2}+g\left(A_{\mu}+\widetilde{C}_{\mu}\right) X_{\nu}^{1}\right\} \hat{n}_{2},
\end{gathered}
$$

where now the magnetic potential $\widetilde{C}_{\mu}$ can be written explicitly as

$$
\widetilde{C}_{\mu}=-\frac{1}{g} \vec{n}_{1} \cdot \partial_{\mu} \vec{n}_{2},
$$

up to the $U(1)$ gauge transformation which leaves $\hat{n}$ invariant. So with

$$
\begin{gathered}
\bar{A}_{\mu}=A_{\mu}+\widetilde{C}_{\mu}, \quad \bar{F}_{\mu \nu}=\partial_{\mu} \bar{A}_{\nu}-\partial_{\nu} \bar{A}_{\mu}, \\
X_{\mu}=\frac{1}{\sqrt{2}}\left(X_{\mu}^{1}+i X_{\mu}^{2}\right),
\end{gathered}
$$

one could express the Lagrangian explicitly in terms of the dual potential $B_{\mu}$ and the complex vector field $X_{\mu}$,

$$
\begin{gathered}
\mathcal{L}=-\frac{1}{4} \bar{F}_{\mu \nu}^{2}-\frac{1}{2}\left|\bar{D}_{\mu} X_{\nu}-\bar{D}_{\nu} X_{\mu}\right|^{2}+i g \bar{F}_{\mu \nu} X_{\mu}^{*} X_{\nu} \\
-\frac{1}{2} g^{2}\left\{\left(X_{\mu}^{*} X_{\mu}\right)^{2}-\left(X_{\mu}^{*}\right)^{2}\left(X_{\nu}\right)^{2}\right\},
\end{gathered}
$$

where now

$$
\bar{D}_{\mu}=\partial_{\mu}+i g \bar{A}_{\mu}
$$

Clearly this describes an Abelian gauge theory coupled to the charged vector field $X_{\mu}$. But the important point here is that the Abelian potential $\bar{A}_{\mu}$ is given by the sum of the electric and magnetic potentials $A_{\mu}+\widetilde{C}_{\mu}$. In this form the equations of motion (32) is re-expressed as

$$
\begin{gathered}
\partial_{\mu}\left(\bar{F}_{\mu \nu}+X_{\mu \nu}\right)=i g X_{\mu}^{*}\left(\bar{D}_{\mu} X_{\nu}-\bar{D}_{\nu} X_{\mu}\right) \\
-i g X_{\mu}\left(\bar{D}_{\mu} X_{\nu}-\bar{D}_{\nu} X_{\mu}\right)^{*} \\
\bar{D}_{\mu}\left(\bar{D}_{\mu} X_{\nu}-\bar{D}_{\nu} X_{\mu}\right)=i g X_{\mu}\left(\bar{F}_{\mu \nu}+X_{\mu \nu}\right) .
\end{gathered}
$$

where now

$$
X_{\mu \nu}=-i g\left(X_{\mu}^{*} X_{\nu}-X_{\nu}^{*} X_{\mu}\right)
$$

This shows that one can indeed Abelianize the nonAbelian theory with our decomposition. The remarkable change in this "Abelian" formulation is that here the topological field $\hat{n}$ is replaced by the magnetic potential $\widetilde{C}_{\mu}$.

One might ask whether this "Abelian" theory retains the original non-Abelian gauge symmetry, and if so, how the non-Abelian gauge symmetry is realized in this "Abelian" theory. To answer this notice that here we have never fixed the gauge to obtain this Abelian formalism, so that the original non-Abelian gauge symmetry must remain intact. To see this let

$$
\begin{gathered}
\vec{\alpha}=\alpha_{1} \hat{n}_{1}+\alpha_{2} \hat{n}_{2}+\theta \hat{n}, \\
\alpha=\frac{1}{\sqrt{2}}\left(\alpha_{1}+i \alpha_{2}\right), \\
\vec{C}_{\mu}=-\frac{1}{g} \hat{n} \times \partial_{\mu} \hat{n}=-C_{\mu}^{1} \hat{n}_{1}-C_{\mu}^{2} \hat{n}_{2}, \\
C_{\mu}=\frac{1}{\sqrt{2}}\left(C_{\mu}^{1}+i C_{\mu}^{2}\right) .
\end{gathered}
$$


Then the Lagrangian (38) is invariant not only under the active gauge transformation (24) described by

$$
\begin{gathered}
\delta A_{\mu}=\frac{1}{g} \partial_{\mu} \theta-i\left(C_{\mu}^{*} \alpha-C_{\mu} \alpha^{*}\right), \quad \delta \widetilde{C}_{\mu}=-\delta A_{\mu}, \\
\delta X_{\mu}=0,
\end{gathered}
$$

but also under the passive gauge transformation (33) described by

$$
\begin{gathered}
\delta A_{\mu}=\frac{1}{g} \partial_{\mu} \theta-i\left(X_{\mu}^{*} \alpha-X_{\mu} \alpha^{*}\right), \quad \delta \widetilde{C}_{\mu}=0, \\
\delta X_{\mu}=\frac{1}{g} \bar{D}_{\mu} \alpha-i \theta X_{\mu} .
\end{gathered}
$$

This tells that the "Abelian" theory not only retains the original non-Abelian gauge symmetry, but actually has an enlarged (both the active and passive) gauge symmetries. Again this is because this Abelianization is not the "naive" Abelianization of the $S U(2)$ gauge theory which one obtains by fixing the gauge. Our Abelianization is a gauge independent Abelianization which retains the full non-Abelian gauge symmetry of the original theory. Moreover, we emphasize that here the Abelian gauge group is actually made of $U(1)_{e} \otimes U(1)_{m}$, so that the Abelian theory becomes a dual gauge theory [12, 15]. This is evident from (41) and (42).

The above $U(1)$ decomposition of $S U(2)$ was first introduced long time ago in an attempt to demonstrate the monopole condensation and color confinement in QCD [12, 13], and the importance of the decomposition in establishing the Abelian dominance in non-Abelian dynamics has become appreciated by many authors [14, 17]. Now we show that this decomposition plays a crucial role for us to make the Abelian decomposition of the Einstein's theory.

\section{DECOMPOSITION OF GRAVITATIONAL CONNECTION}

We apply the above Abelian decomposition to Einstein's theory, regarding Einstein's theory as a gauge theory of Lorentz group. To do this we introduce a coordinate basis

$$
\left[\partial_{\mu}, \partial_{\nu}\right]=0, \quad(\mu, \nu=t, x, y, z)
$$

and an orthonormal basis

$$
\begin{aligned}
& {\left[\xi_{a}, \xi_{b}\right]=f_{a b}{ }^{c} \xi_{c} . \quad(a, b=0,1,2,3)} \\
& \xi_{a}=e_{a}^{\mu} \partial_{\mu}, \quad \partial_{\mu}=e_{\mu}^{a} \xi_{a},
\end{aligned}
$$

where $e_{\mu}^{a}$ and $e_{a}^{\mu}$ are the tetrad and inverse tetrad. Let $J_{a b}=-J_{b a}$ be the generators of Lorentz group,

$$
\begin{gathered}
{\left[J_{a b}, J_{c d}\right]=\eta_{a c} J_{b d}-\eta_{b c} J_{a d}+\eta_{b d} J_{a c}-\eta_{a d} J_{b c}} \\
=f_{a b, c d}{ }^{m n} J_{m n}, \\
f_{a b, c d}{ }^{m n}=\eta_{a c} \delta_{b}^{[m} \delta_{d}^{n]}-\eta_{b c} \delta_{a}^{[m} \delta_{d}^{n]} \\
+\eta_{b d} \delta_{a}^{[m} \delta_{c}{ }^{n]}-\eta_{a d} \delta_{b}^{[m} \delta_{c}{ }^{n]},
\end{gathered}
$$

where $\eta_{a b}=\operatorname{diag}(-1,1,1,1)$ is the Minkowski metric. Clearly $J_{a b}$ has the following 4-dimensional matrix representation

$$
\left(J_{a b}\right)_{c}{ }^{d}=-\eta_{a c} \delta_{b}{ }^{d}+\eta_{b c} \delta_{a}{ }^{d},
$$

so that under the infinitesimal gauge transformation we have

$$
\delta e_{\mu}^{c}=\left(\eta_{a d} \delta_{b}^{c}-\eta_{b d} \delta_{a}^{c}\right) \alpha^{a b} e_{\mu}^{d},
$$

where $\alpha^{a b}\left(=-\alpha^{b a}\right)$ is an infinitesimal gauge parameter of the Lorentz group. Instead of $(a b, c d, \ldots)$ we can use the index $(A, B, \ldots)=(1,2,3,4,5,6)=(23,31,12,01,02,03)$, and write

$$
\left[J_{A}, J_{B}\right]=f_{A B}{ }^{C} J_{C} .
$$

Moreover, with

$$
\begin{aligned}
& L_{1,2,3}=J_{1,2,3}=J_{23,31,12} \\
& K_{1,2,3}=J_{4,5,6}=J_{01,02,03}
\end{aligned}
$$

the Lorentz algebra is written as

$$
\begin{gathered}
{\left[L_{i}, L_{j}\right]=\epsilon_{i j k} L_{k},} \\
{\left[L_{i}, K_{j}\right]=\epsilon_{i j k} K_{k},} \\
{\left[K_{i}, K_{j}\right]=-\epsilon_{i j k} L_{k}, \quad(i, j, k=1,2,3)}
\end{gathered}
$$

where $L_{i}$ and $K_{i}$ are the 3 -dimensional rotation and boost generators. Notice that the generators can be viewed as the left-invariant basis vector fields on the Lorentz group manifold which satisfy the commutation relation.

As we have pointed out, we can regard Einstein's theory as a gauge theory of Lorentz group. In this view the gravitational connection $\Gamma_{\mu \nu}^{\rho}$ (or more precisely the spin connection $\omega_{\mu}^{a b}$ ) corresponds to the gauge potential $\Gamma_{\mu}^{a b}$, and the curvature tensor $R_{\mu \nu}^{a b}$ corresponds to the gauge field strength $F_{\mu \nu}^{a b}$ of Lorentz group. And to obtain the desired decomposition we have to decompose the gauge potential $\Gamma_{\mu}^{a b}$ first. Now, to apply the above $S U(2)$ decomposition to Lorentz group, we have to keep in mind that there are notable differences between $S U(2)$ and Lorentz group. First, the Lorentz group is non-compact, so that the invariant metric is indefinite. Secondly, the Lorentz group has the well-known invariant tensor $\epsilon_{a b c d}$ which allows the dual transformation. Thirdly, the Lorentz group has rank two, so that it has two commuting Abelian subgroups and two Casimir invariants. Finally, the Lorentz group has two different maximal Abelian subgroups $A_{2}$ and $B_{2}$ [25]. These differences make the decomposition more complicated.

The invariant metric $\delta_{A B}$ of Lorentz group is given by

$$
\begin{gathered}
\delta_{A B}=-\frac{1}{4} f_{A C}^{D} f_{B D}{ }^{C} \\
=\operatorname{diag}(1,1,1,-1,-1,-1) .
\end{gathered}
$$


Let $p^{a b}\left(p^{a b}=-p^{b a}\right)\left(\right.$ or $\left.p^{A}\right)$ be a gauge covariant sextet vector which forms an adjoint representation of Lorentz group,

$$
\delta p^{c d}=-\frac{1}{2} f_{a b, m n}^{c d} \alpha^{a b} p^{m n} .
$$

Clearly it can be understood as an anti-symmetric tensor in 4-dimensional Minkowski space which can be expressed by two 3 -dimensional vectors $\vec{m}$ and $\vec{e}$, which transform exactly like the magnetic and electric components of an electromagnetic tensor under the 4-dimensional Lorentz transformation. And we can denote $p^{a b}$ by $\mathbf{p}$,

$$
\begin{gathered}
\mathbf{p}=\frac{1}{2} p_{a b} \mathbf{I}^{a b}=\left(\begin{array}{c}
\vec{m} \\
\vec{e}
\end{array}\right), \quad p^{a b}=\mathbf{p} \cdot \mathbf{I}^{a b}=\frac{1}{2} p^{m n} I_{m n}{ }^{a b}, \\
\mathbf{I}^{a b}=\left(\begin{array}{c}
\hat{a}^{a b} \\
\hat{b}^{a b}
\end{array}\right), \\
\hat{a}_{i}^{a b}=\epsilon_{0 i}{ }^{a b}, \quad \hat{b}_{i}^{a b}=\left(\delta_{0}{ }^{a} \delta_{i}{ }^{b}-\delta_{0}{ }^{b} \delta_{i}{ }^{a}\right), \\
I_{c d}{ }^{a b}=\left(\delta_{c}{ }^{a} \delta_{d}{ }^{b}-\delta_{c}{ }^{b} \delta_{d}{ }^{a}\right)=-\left(J_{c d}\right)^{a b} .
\end{gathered}
$$

where $m_{i}=\epsilon_{i j k} p^{j k} / 2(i, j, k=1,2,3)$ is the magnetic (or rotation) part and $e_{i}=p^{0 i}$ is the electric (or boost) part of p. From the invariant metric (48) we have

$$
\mathbf{p}^{2}=\frac{1}{2} p_{a b} p^{a b}=\vec{m}^{2}-\vec{e}^{2}
$$

so that the invariant length can be positive, zero, or negative. This, of course, is due to the fact that the invariant metric (48) is indefinite.

The Lorentz group has another important invariant tensor $\epsilon_{A B}$ which comes from the totally anti-symmetric invariant tensor $\epsilon_{a b c d}$,

$$
\epsilon_{A B}=\epsilon_{a b, c d}=\epsilon_{a b c d}
$$

This tells that any adjoint representation of Lorentz group has its dual partner. In particular, $\mathbf{p}$ has the dual vector $\tilde{\mathbf{p}}$ defined by $\widetilde{p}^{a b}=\epsilon^{a b c d} p_{c d} / 2$. With (50) we have (with $\epsilon_{0123}=+1$ )

$$
\begin{gathered}
\tilde{\mathbf{p}}=\left(\begin{array}{c}
\vec{e} \\
-\vec{m}
\end{array}\right), \quad \tilde{\tilde{\mathbf{p}}}=-\mathbf{p}, \\
\tilde{\mathbf{p}}^{2}=\vec{e}^{2}-\vec{m}^{2}=-\mathbf{p}^{2}, \\
\mathbf{p} \cdot \tilde{\mathbf{p}}=\frac{1}{4} \epsilon_{a b c d} p^{a b} p^{c d}=2 \vec{m} \cdot \vec{e} .
\end{gathered}
$$

Moreover, since

$$
\begin{aligned}
& \left(p \times p^{\prime}\right)^{a b}=\left(\mathbf{p} \times \mathbf{p}^{\prime}\right) \cdot \mathbf{I}^{a b} \\
& =-\eta_{c d}\left(p^{a c} p^{\prime b d}-p^{b c} p^{\prime a d}\right),
\end{aligned}
$$

we have

$$
[p, \tilde{p}]=0
$$

or equivalently

$$
\mathbf{p} \times \tilde{\mathbf{p}}=0
$$

This tells that any two vectors which are dual to each other are always commuting. Finally with two vectors $\mathbf{p}$ and $\mathbf{p}^{\prime}$ we have

$$
\begin{gathered}
\mathbf{p} \cdot \mathbf{p}^{\prime}=\vec{m} \cdot \vec{m}^{\prime}-\vec{e} \cdot \vec{e}, \\
\mathbf{p} \cdot \tilde{\mathbf{p}}^{\prime}=\vec{m} \cdot \vec{e}+\vec{e} \cdot \vec{m}^{\prime}=\tilde{\mathbf{p}} \cdot \mathbf{p}^{\prime}, \\
\mathbf{p} \times \mathbf{p}^{\prime}=\left(\begin{array}{c}
\vec{m} \times \vec{m}^{\prime}-\vec{e} \times \vec{e}^{\prime} \\
\vec{m} \times \vec{e}^{\prime}+\vec{e} \times \vec{m}^{\prime}
\end{array}\right)=-\tilde{\mathbf{p}} \times \tilde{\mathbf{p}}^{\prime}, \\
\mathbf{p} \times \tilde{\mathbf{p}}^{\prime}=\left(\begin{array}{c}
\vec{m} \times \vec{e}^{\prime}+\vec{e} \times \vec{m}^{\prime} \\
-\vec{m} \times \vec{m}^{\prime}+\vec{e} \times \vec{e}^{\prime}
\end{array}\right)=\tilde{\mathbf{p}} \times \mathbf{p}^{\prime}, \\
\widetilde{\mathbf{p} \times \mathbf{p}^{\prime}=\mathbf{p} \times \tilde{\mathbf{p}}^{\prime},}
\end{gathered}
$$

so that we can always reduce the operations of sextet vectors of Lorentz group to the operations of 3-dimensional vectors.

Let $\left(\hat{n}_{1}, \hat{n}_{2}, \hat{n}_{3}=\hat{n}\right)$ be a 3 -dimensional unit vectors $\left(\hat{n}_{i}^{2}=1\right)$ which form a right-handed orthonormal basis with $\hat{n}_{1} \times \hat{n}_{2}=\hat{n}_{3}$, and let

$$
\mathbf{l}_{i}=\left(\begin{array}{c}
\hat{n}_{i} \\
0
\end{array}\right), \quad \mathbf{k}_{i}=\left(\begin{array}{c}
0 \\
\hat{n}_{i}
\end{array}\right)=-\tilde{\mathbf{l}}_{i} .
$$

Clearly we have

$$
\begin{aligned}
\mathbf{l}_{i} \cdot \mathbf{l}_{j}=\delta_{i j}, \quad \mathbf{l}_{i} \cdot \mathbf{k}_{j} & =0, \quad \mathbf{k}_{i} \cdot \mathbf{k}_{j}=-\delta_{i j}, \\
\mathbf{l}_{i} \times \mathbf{l}_{j} & =\epsilon_{i j k} \mathbf{l}_{k}, \\
\mathbf{l}_{i} \times \mathbf{k}_{j} & =\epsilon_{i j k} \mathbf{k}_{k}, \\
\mathbf{k}_{i} \times \mathbf{k}_{j} & =-\epsilon_{i j k} \mathbf{l}_{k}
\end{aligned}
$$

so that $\left(\mathbf{l}_{i}, \mathbf{k}_{i}\right)$, or equivalently $\left(\mathbf{l}_{i}, \tilde{\mathbf{l}}_{i}\right)$, forms an orthonormal basis of the adjoint representation of Lorentz group.

To make the desired Abelian decomposition we have to choose the gauge covariant sextet vector fields which form adjoint representation of Lorentz group which describe the desired magnetic isometry. To see what types of isometry is possible, it is important to remember that Lorentz group has two maximal Abelian subgroups, $A_{2}$ made of $L_{3}$ and $K_{3}$ and $B_{2}$ made of $\left(L_{1}+K_{2}\right) / \sqrt{2}$ and $\left(L_{2}-K_{1}\right) / \sqrt{2}[25]$. This tells that we have two possible Abelian decompositions of the gravitational connection. And in both cases the magnetic isometry is described by two, not one, commuting sextet vector fields of Lorentz group which are dual to each other. To see this let us denote one of the isometry vector field by $\mathbf{p}$ which satisfy the isometry condition

$$
D_{\mu} \mathbf{p}=\left(\partial_{\mu}+\boldsymbol{\Gamma}_{\mu} \times\right) \mathbf{p}=0
$$

where we have normalized the coupling constant to be the unit (which one can always do without loss of generality). Now, notice that the above condition automatically assures

$$
D_{\mu} \tilde{\mathbf{p}}=\left(\partial_{\mu}+\boldsymbol{\Gamma}_{\mu} \times\right) \tilde{\mathbf{p}}=0
$$

because $\epsilon_{a b c d}$ is an invariant tensor. This tells that when $\mathbf{p}$ is an isometry, $\tilde{\mathbf{p}}$ also becomes an isometry. To verify 
this directly we decompose the gauge potential of Lorentz group $\boldsymbol{\Gamma}_{\mu}$ into the 3 -dimensional rotation and boost parts $\vec{A}_{\mu}$ and $\vec{B}_{\mu}$, and let

$$
\Gamma_{\mu}=\left(\begin{array}{c}
\vec{A}_{\mu} \\
\vec{B}_{\mu}
\end{array}\right) .
$$

With this both (60) and (61) can be written as

$$
\begin{gathered}
D_{\mu} \vec{m}=\vec{B}_{\mu} \times \vec{e}, \\
D_{\mu} \vec{e}=-\vec{B}_{\mu} \times \vec{m},
\end{gathered}
$$

where now

$$
D_{\mu}=\partial_{\mu}+\vec{A}_{\mu} \times .
$$

This confirms that (60) and (61) are actually identical to each other, which tells that the magnetic isometry in Lorentz group must be even-dimensional.

Since Lorentz group has two invariant tensors it has two Casimir invariants. And it is useful to characterize the isometry by two Casimir invariants. Let the isometry is described by $\mathbf{p}$ and $\tilde{\mathbf{p}}$. It has two Casimir invariants $\alpha$ and $\beta$,

$$
\begin{gathered}
\alpha=\mathbf{p} \cdot \mathbf{p}=\vec{m}^{2}-\vec{e}^{2} \\
\beta=\mathbf{p} \cdot \tilde{\mathbf{p}}=2 \vec{m} \cdot \vec{e} .
\end{gathered}
$$

But the Casimir invariants $(\alpha, \beta)$ depends on the choice of the isometry vectors. To see this consider $\mathbf{p}^{\prime}$ and $\tilde{\mathbf{p}}^{\prime}$ given by a linear combination of $\mathbf{p}$ and $\tilde{\mathbf{p}}$,

$$
\mathbf{p}^{\prime}=a \mathbf{p}+b \tilde{\mathbf{p}}, \quad \tilde{\mathbf{p}}^{\prime}=a \tilde{\mathbf{p}}-b \mathbf{p} .
$$

Clearly we have

$$
D_{\mu} \mathbf{p}^{\prime}=0, \quad D_{\mu} \tilde{\mathbf{p}}^{\prime}=0,
$$

so that they can also be viewed to describe the same isometry. But their Casimir invariants $\left(\alpha^{\prime}, \beta^{\prime}\right)$ are given by

$$
\begin{aligned}
& \alpha^{\prime}=\left(a^{2}-b^{2}\right) \alpha+2 a b \beta \\
& \beta^{\prime}=\left(a^{2}-b^{2}\right) \beta-2 a b \alpha .
\end{aligned}
$$

And with

$$
a^{2}=\frac{\sqrt{\alpha^{2}+\beta^{2}}+|\alpha|}{2\left(\alpha^{2}+\beta^{2}\right)}, \quad b^{2}=\frac{\sqrt{\alpha^{2}+\beta^{2}}-|\alpha|}{2\left(\alpha^{2}+\beta^{2}\right)},
$$

we can always make

$$
\alpha^{\prime}=1, \quad \beta^{\prime}=0,
$$

unless $\alpha^{2}+\beta^{2}=0$. This tells that we can always choose $\mathbf{p}$ and $\tilde{\mathbf{p}}$ in such a way to make $(\alpha, \beta)$ to be $(1,0)$ or $(0,0)$. Physically this means that the magnetic isometry in Einstein's theory can be classified by the space-like (or equivalently time-like) isometry and the light-like isometry whose Casimir invariants are denoted by $(1,0)$ and $(0,0)$, respectively. Notice that there is no need to talk about the time-like isometry, because the isometry vectors always contains the dual partners. But we emphasize that once $\mathbf{p}$ and $\tilde{\mathbf{p}}$ are chosen, $(\alpha, \beta)$ are uniquely fixed. Now we discuss the two isometries $A_{2}$ and $B_{2}$ separately.

\section{A. $A_{2}$ (Space-like) Isometry}

Let the maximal Abelian subgroup be $A_{2}$. In this case the isometry is made of $L_{3}$ and $K_{3}$, and we have two sextet vector fields which describes the isometry which are dual to each other. Let $\mathbf{p}$ and $\tilde{\mathbf{p}}$ be the two isometry vector fields which correspond to $L_{3}$ and $K_{3}$. Clearly we can put

$$
\begin{gathered}
\mathbf{p}=f \mathbf{l}_{3}=f\left(\begin{array}{c}
\hat{n} \\
0
\end{array}\right), \\
\tilde{\mathbf{p}}=f \tilde{\mathbf{l}}_{3}=f\left(\begin{array}{c}
0 \\
-\hat{n}
\end{array}\right),
\end{gathered}
$$

where $f$ is an arbitrary function of space-time. The Casimir invariants of the isometry vectors are given by $\left(f^{2}, 0\right)$. But just as in $S U(2)$ gauge theory the isometry condition (60) requires $f$ to be a constant, because

$$
\partial_{\mu} f^{2}=\partial_{\mu} \mathbf{p}^{2}=2 \mathbf{p} \cdot D_{\mu} \mathbf{p}=0 .
$$

And we can always normalize $f=1$ without loss of generality.

So the $A_{2}$ isometry can always be written as

$$
\begin{gathered}
\mathbf{l}=\mathbf{l}_{3}=\left(\begin{array}{c}
\hat{n} \\
0
\end{array}\right), \quad \tilde{\mathbf{l}}=\tilde{\mathbf{l}}_{3}=\left(\begin{array}{c}
0 \\
-\hat{n}
\end{array}\right), \\
D_{\mu} \mathbf{l}=0, \quad D_{\mu} \tilde{\mathbf{l}}=0,
\end{gathered}
$$

whose Casimir invariants are fixed by $(1,0)$. With this we find the restricted connection $\hat{\boldsymbol{\Gamma}}_{\mu}$ which satisfies the isometry condition

$$
\begin{gathered}
\hat{\mathbf{\Gamma}}_{\mu}=\Gamma_{\mu} \mathbf{l}-\widetilde{\Gamma}_{\mu} \tilde{\mathbf{l}}-\mathbf{l} \times \partial_{\mu} \mathbf{l}, \\
\Gamma_{\mu}=\mathbf{l} \cdot \boldsymbol{\Gamma}_{\mu}, \quad \widetilde{\Gamma}_{\mu}=\tilde{\mathbf{l}} \cdot \boldsymbol{\Gamma}_{\mu},
\end{gathered}
$$

where $\Gamma_{\mu}$ and $\widetilde{\Gamma}_{\mu}$ are two Abelian connections of $\mathbf{l}$ and $\tilde{\mathbf{l}}$ components which are not restricted by the isometry condition. At first glance this expression appears strange, because one expects that $\mathbf{l}$ and $\tilde{\mathbf{l}}$ should contribute equally in the restricted connection since (60) and (61) are identical. Actually they do contribute equally because we have

$$
\mathbf{l} \times \partial_{\mu} \mathbf{l}=-\tilde{\mathbf{l}} \times \partial_{\mu} \tilde{\mathbf{l}},
$$

so that we can express the restricted connection as

$$
\hat{\boldsymbol{\Gamma}}_{\mu}=\Gamma_{\mu} \mathbf{l}-\widetilde{\Gamma}_{\mu} \tilde{\mathbf{l}}-\frac{1}{2}\left(\mathbf{l} \times \partial_{\mu} \mathbf{l}-\tilde{\mathbf{l}} \times \partial_{\mu} \tilde{\mathbf{l}}\right) .
$$

The restricted field strength $\hat{\mathbf{R}}_{\mu \nu}$ which describes the restricted curvature tensor $\hat{R}_{\mu \nu}^{a b}$ is given by

$$
\begin{gathered}
\hat{\mathbf{R}}_{\mu \nu}=\partial_{\mu} \hat{\boldsymbol{\Gamma}}_{\nu}-\partial_{\nu} \hat{\mathbf{\Gamma}}_{\mu}+\hat{\boldsymbol{\Gamma}}_{\mu} \times \hat{\mathbf{\Gamma}}_{\nu} \\
=\left(\Gamma_{\mu \nu}+H_{\mu \nu}\right) \mathbf{l}-\left(\widetilde{\Gamma}_{\mu \nu}+\widetilde{H}_{\mu \nu}\right) \tilde{\mathbf{l}}, \\
\Gamma_{\mu \nu}=\partial_{\mu} \Gamma_{\nu}-\partial_{\nu} \Gamma_{\mu}, \quad \widetilde{\Gamma}_{\mu \nu}=\partial_{\mu} \widetilde{\Gamma}_{\nu}-\partial_{\nu} \widetilde{\Gamma}_{\mu}, \\
H_{\mu \nu}=-\mathbf{l} \cdot\left(\partial_{\mu} \mathbf{l} \times \partial_{\nu} \mathbf{l}\right), \\
\widetilde{H}_{\mu \nu}=-\tilde{\mathbf{l}} \cdot\left(\partial_{\mu} \mathbf{l} \times \partial_{\nu} \mathbf{l}\right)=\tilde{\mathbf{l}} \cdot\left(\partial_{\mu} \tilde{\mathbf{l}} \times \partial_{\nu} \tilde{\mathbf{l}}\right) \\
=0,
\end{gathered}
$$


so that we have

$$
\begin{gathered}
\hat{R}_{\mu \nu}^{a b}=\hat{\mathbf{R}}_{\mu \nu} \cdot \mathbf{I}^{a b} \\
=\left(\Gamma_{\mu \nu}+H_{\mu \nu}\right) l^{a b}-\widetilde{\Gamma}_{\mu \nu} \tilde{l}^{a b} .
\end{gathered}
$$

Notice that $\widetilde{H}_{\mu \nu}$ vanishes.

In 3-dimensional notation the isometry condition (71) can be written as

$$
\begin{gathered}
\hat{\boldsymbol{\Gamma}}_{\mu}=\left(\begin{array}{c}
\hat{A}_{\mu} \\
\hat{B}_{\mu}
\end{array}\right), \\
\hat{D}_{\mu} \hat{n}=0, \quad \hat{B}_{\mu} \times \hat{n}=0, \\
\hat{D}_{\mu}=\partial_{\mu}+\hat{A}_{\mu} \times .
\end{gathered}
$$

From this we have

$$
\begin{gathered}
\hat{A}_{\mu}=\Gamma_{\mu} \hat{n}-\hat{n} \times \partial_{\mu} \hat{n}, \quad \hat{B}_{\mu}=\widetilde{\Gamma}_{\mu} \hat{n}, \\
\Gamma_{\mu}=\hat{n} \cdot \hat{A}_{\mu}, \quad \widetilde{\Gamma}_{\mu}=\hat{n} \cdot \hat{B}_{\mu} .
\end{gathered}
$$

Moreover, with

$$
\hat{\mathbf{R}}_{\mu \nu}=\left(\begin{array}{c}
\hat{A}_{\mu \nu} \\
\hat{B}_{\mu \nu}
\end{array}\right)
$$

we have

$$
\begin{gathered}
\hat{A}_{\mu \nu}=\partial_{\mu} \hat{A}_{\nu}-\partial_{\nu} \hat{A}_{\mu}+\hat{A}_{\mu} \times \hat{A}_{\nu}-\hat{B}_{\mu} \times \hat{B}_{\nu} \\
=\left(\Gamma_{\mu \nu}+H_{\mu \nu}\right) \hat{n}, \\
\hat{B}_{\mu \nu}=\partial_{\mu} \hat{B}_{\nu}-\partial_{\nu} \hat{B}_{\mu}+\hat{A}_{\mu} \times \hat{B}_{\nu}+\hat{B}_{\mu} \times \hat{A}_{\nu} \\
=\hat{D}_{\mu} \hat{B}_{\nu}-\hat{D}_{\nu} \hat{B}_{\mu}=\widetilde{\Gamma}_{\mu \nu} \hat{n}, \\
H_{\mu \nu}=-\hat{n} \cdot\left(\partial_{\mu} \hat{n} \times \partial_{\nu} \hat{n}\right)=\partial_{\mu} \widetilde{C}_{\nu}-\partial_{\nu} \widetilde{C}_{\mu}, \\
\widetilde{C}_{\mu}=-\hat{n}_{1} \cdot \partial_{\mu} \hat{n}_{2} .
\end{gathered}
$$

Notice that $\hat{A}_{\mu}$ and $\hat{A}_{\mu \nu}$ are formally identical to the restricted potential and restricted field strength of $S U(2)$ gauge theory. In particular $H_{\mu \nu}$ is identical to what we have in Section III. This, together with $\widetilde{H}_{\mu \nu}=0$, tells that the topology of this isometry is identical to that of the $S U(2)$ subgroup. by

With this the full connection of Lorentz group is given

$$
\boldsymbol{\Gamma}_{\mu}=\hat{\boldsymbol{\Gamma}}_{\mu}+\mathbf{Z}_{\mu}, \quad \mathbf{l} \cdot \mathbf{Z}_{\mu}=\tilde{\mathbf{l}} \cdot \mathbf{Z}_{\mu}=0
$$

where $\mathbf{Z}_{\mu}$ is the valence connection which transforms covariantly under the Lorentz gauge transformation, or equivalently under the general coordinate transformation. The corresponding field strength $\mathbf{R}_{\mu \nu}$ which describes the curvature tensor is written as

$$
\begin{gathered}
\mathbf{R}_{\mu \nu}=\partial_{\mu} \boldsymbol{\Gamma}_{\nu}-\partial_{\nu} \boldsymbol{\Gamma}_{\mu}+\boldsymbol{\Gamma}_{\mu} \times \boldsymbol{\Gamma}_{\nu} \\
=\hat{\mathbf{R}}_{\mu \nu}+\mathbf{Z}_{\mu \nu} \\
\mathbf{Z}_{\mu \nu}=\hat{D}_{\mu} \mathbf{Z}_{\nu}-\hat{D}_{\nu} \mathbf{Z}_{\mu}+\mathbf{Z}_{\mu} \times \mathbf{Z}_{\nu} \\
\hat{D}_{\mu}=\partial_{\mu}+\hat{\boldsymbol{\Gamma}}_{\mu} \times
\end{gathered}
$$

where $\mathbf{Z}_{\mu \nu}$ is the valence part of the curvature tensor which can further be decomposed to the kinetic part $\dot{\mathbf{Z}}_{\mu \nu}$ and the potential part $\mathbf{Z}_{\mu \nu}^{\prime}$,

$$
\begin{gathered}
\mathbf{Z}_{\mu \nu}=\dot{\mathbf{Z}}_{\mu \nu}+\mathbf{Z}_{\mu \nu}^{\prime} \\
\dot{\mathbf{Z}}_{\mu \nu}=\hat{D}_{\mu} \mathbf{Z}_{\nu}-\hat{D}_{\nu} \mathbf{Z}_{\mu}, \quad \mathbf{Z}_{\mu \nu}^{\prime}=\mathbf{Z}_{\mu} \times \mathbf{Z}_{\nu}
\end{gathered}
$$

Now with

$$
\begin{gathered}
\mathbf{Z}_{\mu}=Z_{\mu}^{1} \mathbf{l}_{1}-\widetilde{Z}_{\mu}^{1} \tilde{\mathbf{l}}_{1}+Z_{\mu}^{2} \mathbf{l}_{2}-\widetilde{Z}_{\mu}^{2} \tilde{\mathbf{l}}_{2}, \\
Z_{\mu}^{1}=\mathbf{l}_{1} \cdot \mathbf{Z}_{\mu}, \quad \widetilde{Z}_{\mu}^{1}=\tilde{\mathbf{l}}_{1} \cdot \mathbf{Z}_{\mu} \\
Z_{\mu}^{2}=\mathbf{l}_{2} \cdot \mathbf{Z}_{\mu}, \quad \widetilde{Z}_{\mu}^{2}=\tilde{\mathbf{l}}_{2} \cdot \mathbf{Z}_{\mu}
\end{gathered}
$$

we have

$$
\begin{gathered}
\dot{\mathbf{Z}}_{\mu \nu}=\left(\mathcal{D}_{\mu} Z_{\nu}^{1}-\mathcal{D}_{\nu} Z_{\mu}^{1}\right) \mathbf{l}_{1}-\left(\mathcal{D}_{\mu} \widetilde{Z}_{\nu}^{1}-\mathcal{D}_{\nu} \widetilde{Z}_{\mu}^{1}\right) \tilde{\mathbf{l}}_{1} \\
+\left(\mathcal{D}_{\mu} Z_{\nu}^{2}-\mathcal{D}_{\nu} Z_{\mu}^{2}\right) \mathbf{l}_{2}-\left(\mathcal{D}_{\mu} \widetilde{Z}_{\nu}^{2}-\mathcal{D}_{\nu} \widetilde{Z}_{\mu}^{2}\right) \tilde{\mathbf{l}}_{2} \\
\mathcal{D}_{\mu} Z_{\nu}^{1}=\partial_{\mu} Z_{\nu}^{1}-L_{\mu} Z_{\nu}^{2}-\widetilde{L}_{\mu} \widetilde{Z}_{\nu}^{2} \\
\mathcal{D}_{\mu} \widetilde{Z}_{\nu}^{1}=\partial_{\mu} \widetilde{Z}_{\nu}^{1}-L_{\mu} \widetilde{Z}_{\nu}^{2}+\widetilde{L}_{\mu} Z_{\nu}^{2} \\
\mathcal{D}_{\mu} Z_{\nu}^{2}=\partial_{\mu} Z_{\nu}^{2}+L_{\mu} Z_{\nu}^{1}-\widetilde{L}_{\mu} \widetilde{Z}_{\nu}^{1} \\
\mathcal{D}_{\mu} \widetilde{Z}_{\nu}^{2}=\partial_{\mu} \widetilde{Z}_{\nu}^{2}+L_{\mu} \widetilde{Z}_{\nu}^{1}+\widetilde{L}_{\mu} Z_{\nu}^{1} \\
L_{\mu}=\Gamma_{\mu}+\widetilde{C}_{\mu}, \quad \widetilde{L}_{\mu}=\widetilde{\Gamma}_{\mu} \\
\mathbf{l} \cdot \dot{\mathbf{Z}}_{\mu \nu}=\tilde{\mathbf{l}} \cdot \dot{\mathbf{Z}}_{\mu \nu}=0
\end{gathered}
$$

Clearly $L_{\mu}$ is identical to the dual potential we have introduced in Section III in $S U(2)$ gauge theory. Moreover, we have

$$
\begin{gathered}
\mathbf{Z}_{\mu \nu}^{\prime}=W_{\mu \nu} \mathbf{l}-\widetilde{W}_{\mu \nu} \tilde{\mathbf{l}}, \\
W_{\mu \nu}=\mathbf{l} \cdot\left(\mathbf{Z}_{\mu} \times \mathbf{Z}_{\nu}\right) \\
=Z_{\mu}^{1} Z_{\nu}^{2}-Z_{\nu}^{1} Z_{\mu}^{2}-\widetilde{Z}_{\mu}^{1} \widetilde{Z}_{\nu}^{2}+\widetilde{Z}_{\nu}^{1} \widetilde{Z}_{\mu}^{2}, \\
\widetilde{W}_{\mu \nu}=\tilde{\mathbf{l}} \cdot\left(\mathbf{Z}_{\mu} \times \mathbf{Z}_{\nu}\right) \\
=Z_{\mu}^{1} \widetilde{Z}_{\nu}^{2}-Z_{\nu}^{1} \widetilde{Z}_{\mu}^{2}+\widetilde{Z}_{\mu}^{1} Z_{\nu}^{2}-\widetilde{Z}_{\nu}^{1} Z_{\mu}^{2} .
\end{gathered}
$$

With this we have the full curvature tensor

$$
\begin{gathered}
\mathbf{R}_{\mu \nu}=\left(\Gamma_{\mu \nu}+H_{\mu \nu}+W_{\mu \nu}\right) \mathbf{l}-\left(\widetilde{\Gamma}_{\mu \nu}+\widetilde{W}_{\mu \nu}\right) \mathbf{l} \\
\quad+\hat{D}_{\mu} \mathbf{Z}_{\nu}-\hat{D}_{\nu} \mathbf{Z}_{\mu \nu} \\
=\left(\mathcal{D}_{\mu} L_{\nu}-\mathcal{D}_{\nu} L_{\mu}\right) \mathbf{l}-\left(\mathcal{D}_{\mu} \widetilde{L}_{\nu}-\mathcal{D}_{\nu} \widetilde{L}_{\mu}\right) \tilde{\mathbf{l}} \\
+\left(\mathcal{D}_{\mu} Z_{\nu}^{1}-\mathcal{D}_{\nu} Z_{\mu}^{1}\right) \mathbf{l}_{1}-\left(\mathcal{D}_{\mu} \widetilde{Z}_{\nu}^{1}-\mathcal{D}_{\nu} \widetilde{Z}_{\mu}^{1}\right) \tilde{\mathbf{l}}_{1} \\
+\left(\mathcal{D}_{\mu} Z_{\nu}^{2}-\mathcal{D}_{\nu} Z_{\mu}^{2}\right) \mathbf{l}_{2}-\left(\mathcal{D}_{\mu} \widetilde{Z}_{\nu}^{2}-\mathcal{D}_{\nu} \widetilde{Z}_{\mu}^{2}\right) \tilde{\mathbf{l}}_{2} \\
=L_{\mu \nu}^{1} \mathbf{l}_{1}-\widetilde{L}_{\mu \nu}^{1} \tilde{\mathbf{l}}_{1}+L_{\mu \nu}^{2} \mathbf{l}_{2}-\widetilde{L}_{\mu \nu}^{2} \tilde{\mathbf{l}}_{2} \\
\quad+L_{\mu \nu} \mathbf{l}-\widetilde{L}_{\mu \nu} \tilde{\mathbf{l}}, \\
\mathcal{D}_{\mu} L_{\nu}=\partial_{\mu} L_{\nu}+Z_{\mu}^{1} Z_{\nu}^{2}-\widetilde{Z}_{\mu}^{1} \widetilde{Z}_{\nu}^{2}, \\
\mathcal{D}_{\mu} \widetilde{L}_{\nu}=\partial_{\mu} \widetilde{L}_{\nu}-Z_{\mu}^{1} \widetilde{Z}_{\nu}^{2}-\widetilde{Z}_{\mu}^{1} Z_{\nu}^{2}, \\
L_{\mu \nu}^{1}=\mathcal{D}_{\mu} Z_{\nu}^{1}-\mathcal{D}_{\nu} Z_{\mu}^{1}, \quad \widetilde{L}_{\mu \nu}^{1}=\mathcal{D}_{\mu} \widetilde{Z}_{\nu}^{1}-\mathcal{D}_{\nu} \widetilde{Z}_{\mu}^{1}, \\
L_{\mu \nu}^{2}=\mathcal{D}_{\mu} Z_{\nu}^{2}-\mathcal{D}_{\nu} Z_{\mu}^{2}, \quad \widetilde{L}_{\mu \nu}^{2}=\mathcal{D}_{\mu} \widetilde{Z}_{\nu}^{2}-\mathcal{D}_{\nu} \widetilde{Z}_{\mu}^{2}, \\
L_{\mu \nu}=\mathcal{D}_{\mu} L_{\nu}-\mathcal{D}_{\nu} L_{\mu}=\Gamma_{\mu \nu}+H_{\mu \nu}+W_{\mu \nu}, \\
\widetilde{L}_{\mu \nu}=\mathcal{D}_{\mu} \widetilde{L}_{\nu}-\mathcal{D}_{\nu} \widetilde{L}_{\mu}=\widetilde{\Gamma}_{\mu \nu}+\widetilde{W}_{\mu \nu},
\end{gathered}
$$


or equivalently

$$
\begin{gathered}
R_{\mu \nu}^{a b}=\mathbf{R}_{\mu \nu} \cdot \mathbf{I}^{a b} \\
=L_{\mu \nu}^{1} l_{1}^{a b}-\widetilde{L}_{\mu \nu}^{1} \tilde{l}_{1}^{a b}+L_{\mu \nu}^{2} l_{2}^{a b}-\widetilde{L}_{\mu \nu}^{2} \tilde{l}_{2}^{a b} \\
+L_{\mu \nu} l^{a b}-\widetilde{L}_{\mu \nu} \tilde{l}^{a b} .
\end{gathered}
$$

This is the $A_{2}$ decomposition of the curvature tensor. The similarity between this decomposition and the Abelian decomposition of $S U(2)$ is unmistakable.

To emphasize the similarity between this isometry and the $U(1)$ isometry of $S U(2)$ we introduce the complex notation

$$
\begin{array}{cc}
Z_{\mu}=\frac{1}{\sqrt{2}}\left(Z_{\mu}^{1}+i Z_{\mu}^{2}\right), & \widetilde{Z}_{\mu}=\frac{1}{\sqrt{2}}\left(\widetilde{Z}_{\mu}^{1}+i \widetilde{Z}_{\mu}^{2}\right), \\
\mathbf{l}_{ \pm}=\frac{1}{\sqrt{2}}\left(\mathbf{l}_{1} \pm i \mathbf{l}_{2}\right), & \tilde{\mathbf{l}}_{ \pm}=\frac{1}{\sqrt{2}}\left(\tilde{\mathbf{l}}_{1} \pm i \tilde{\mathbf{l}}_{2}\right)
\end{array}
$$

and find

$$
\begin{gathered}
\dot{\mathbf{Z}}_{\mu \nu}=\left(\mathcal{D}_{\mu} Z_{\nu}-\mathcal{D}_{\nu} Z_{\mu}\right)^{*} \mathbf{l}_{+}+\left(\mathcal{D}_{\mu} Z_{\nu}-\mathcal{D}_{\nu} Z_{\mu}\right) \mathbf{l}_{-} \\
-\left(\mathcal{D}_{\mu} \widetilde{Z}_{\nu}-\mathcal{D}_{\nu} \widetilde{Z}_{\mu}\right)^{*} \tilde{\mathbf{l}}_{+}-\left(\mathcal{D}_{\mu} \widetilde{Z}_{\nu}-\mathcal{D}_{\nu} \widetilde{Z}_{\mu}\right) \tilde{\mathbf{l}}_{-} \\
\mathcal{D}_{\mu} Z_{\nu}=\left(\partial_{\mu}+i L_{\mu}\right) Z_{\nu}-i \widetilde{L}_{\mu} \widetilde{Z}_{\nu}^{*}=\bar{D}_{\mu} Z_{\nu}-i \widetilde{L}_{\mu} \widetilde{Z}_{\nu}^{*} \\
\bar{D}_{\mu}=\partial_{\mu}+i L_{\mu}
\end{gathered}
$$

Here $\bar{D}_{\mu}$ is identical to the one we have in Section III. Moreover, the potential part of $\mathbf{Z}_{\mu \nu}$ is given by

$$
\begin{gathered}
\mathbf{Z}_{\mu \nu}^{\prime}=W_{\mu \nu} \mathbf{l}-\widetilde{W}_{\mu \nu} \tilde{\mathbf{l}} \\
W_{\mu \nu}=Z_{\mu}^{1} Z_{\nu}^{2}-Z_{\nu}^{1} Z_{\mu}^{2}-\widetilde{Z}_{\mu}^{1} \widetilde{Z}_{\nu}^{2}+\widetilde{Z}_{\nu}^{1} \widetilde{Z}_{\mu}^{2} \\
=-i\left(Z_{\mu}^{*} Z_{\nu}-Z_{\nu}^{*} Z_{\mu}-\widetilde{Z}_{\mu}^{*} \widetilde{Z}_{\nu}+\widetilde{Z}_{\nu}^{*} \widetilde{Z}_{\mu}\right), \\
\widetilde{W}_{\mu \nu}=Z_{\mu}^{1} \widetilde{Z}_{\nu}^{2}-Z_{\nu}^{1} \widetilde{Z}_{\mu}^{2}+\widetilde{Z}_{\mu}^{1} Z_{\nu}^{2}-\widetilde{Z}_{\nu}^{1} Z_{\mu}^{2} \\
=-i\left(Z_{\mu}^{*} \widetilde{Z}_{\nu}-Z_{\nu}^{*} \widetilde{Z}_{\mu}-\widetilde{Z}_{\mu}^{*} Z_{\nu}+\widetilde{Z}_{\nu}^{*} Z_{\mu}\right) .
\end{gathered}
$$

With this we have

$$
\begin{aligned}
\mathbf{R}_{\mu \nu} & =\left(\mathcal{D}_{\mu} Z_{\nu}-\mathcal{D}_{\nu} Z_{\mu}\right)^{*} \mathbf{l}_{+}-\left(\mathcal{D}_{\mu} \widetilde{Z}_{\nu}-\mathcal{D}_{\nu} \widetilde{Z}_{\mu}\right)^{*} \tilde{\mathbf{l}}_{+} \\
& +\left(\mathcal{D}_{\mu} Z_{\nu}-\mathcal{D}_{\nu} Z_{\mu}\right) \mathbf{l}_{-}-\left(\mathcal{D}_{\mu} \widetilde{Z}_{\nu}-\mathcal{D}_{\nu} \widetilde{Z}_{\mu}\right) \tilde{\mathbf{l}}_{-} \\
& +\left(\mathcal{D}_{\mu} L_{\nu}-\mathcal{D}_{\nu} L_{\mu}\right) \mathbf{l}-\left(\mathcal{D}_{\mu} \widetilde{L}_{\nu}-\mathcal{D}_{\nu} \widetilde{L}_{\mu}\right) \tilde{\mathbf{l}}
\end{aligned}
$$

or

$$
\begin{aligned}
& R_{\mu \nu}^{a b}=\left(\mathcal{D}_{\mu} Z_{\nu}-\mathcal{D}_{\nu} Z_{\mu}\right)^{*} l_{+}^{a b}-\left(\mathcal{D}_{\mu} \widetilde{Z}_{\nu}-\mathcal{D}_{\nu} \widetilde{Z}_{\mu}\right)^{*} \tilde{l}_{+}^{a b} \\
& \quad+\left(\mathcal{D}_{\mu} Z_{\nu}-\mathcal{D}_{\nu} Z_{\mu}\right) l_{-}^{a b}-\left(\mathcal{D}_{\mu} \widetilde{Z}_{\nu}-\mathcal{D}_{\nu} \widetilde{Z}_{\mu}\right) \tilde{l}_{-}^{a b} \\
& \quad+\left(\mathcal{D}_{\mu} L_{\nu}-\mathcal{D}_{\nu} L_{\mu}\right) l^{a b}-\left(\mathcal{D}_{\mu} \widetilde{L}_{\nu}-\mathcal{D}_{\nu} \widetilde{L}_{\mu}\right) \tilde{l}^{a b}
\end{aligned}
$$

This should be compared with the $S U(2)$ decomposition.

In 3-dimensional notation we have

$$
\begin{gathered}
\mathbf{Z}_{\mu}=\left(\begin{array}{c}
\vec{X}_{\mu} \\
\vec{Y}_{\mu}
\end{array}\right), \\
\vec{X}_{\mu}=Z_{\mu}^{1} \hat{n}_{1}+Z_{\mu}^{2} \hat{n}_{2}, \quad \vec{Y}_{\mu}=\widetilde{Z}_{\mu}^{1} \hat{n}_{1}+\widetilde{Z}_{\mu}^{2} \hat{n}_{2}, \\
\hat{n} \cdot \vec{X}_{\mu}=0, \quad \hat{n} \cdot \vec{Y}_{\mu}=0 .
\end{gathered}
$$

Moreover, with

$$
\mathbf{Z}_{\mu \nu}=\left(\begin{array}{c}
\vec{X}_{\mu \nu} \\
\vec{Y}_{\mu \nu}
\end{array}\right)=\left(\begin{array}{c}
\dot{\vec{X}}_{\mu \nu}+\vec{X}_{\mu \nu}^{\prime} \\
\dot{\vec{Y}}_{\mu \nu}+\vec{Y}_{\mu \nu}^{\prime}
\end{array}\right),
$$

we have

$$
\begin{gathered}
\dot{\vec{X}}_{\mu \nu}=\hat{D}_{\mu} \vec{X}_{\nu}-\hat{D}_{\nu} \vec{X}_{\mu}-\vec{B}_{\mu} \times \vec{Y}_{\nu}+\vec{B}_{\nu} \times \vec{Y}_{\mu} \\
=L_{\mu \nu}^{1} \hat{n}_{1}+L_{\mu \nu}^{2} \hat{n}_{2}, \\
\dot{\vec{Y}}_{\mu \nu}=\hat{D}_{\mu} \vec{Y}_{\nu}-\hat{D}_{\nu} \vec{Y}_{\mu}-\vec{B}_{\mu} \times \vec{X}_{\nu}-\vec{B}_{\nu} \times \vec{X}_{\mu} \\
=\widetilde{L}_{\mu \nu}^{1} \hat{n}_{1}+\widetilde{L}_{\mu \nu}^{2} \hat{n}_{2}, \\
\vec{X}_{\mu \nu}^{\prime}=\vec{X}_{\mu} \times \vec{X}_{\nu}-\vec{Y}_{\mu} \times \vec{Y}_{\nu}=W_{\mu \nu} \hat{n}, \\
\vec{Y}_{\mu \nu}^{\prime}=\vec{X}_{\mu} \times \vec{Y}_{\nu}+\vec{Y}_{\mu} \times \vec{X}_{\nu}=\widetilde{W}_{\mu \nu} \hat{n} .
\end{gathered}
$$

Notice that the kinetic part and the potential part of $\mathbf{Z}_{\mu \nu}$ are orthogonal to each other. Finally, with

$$
\mathbf{R}_{\mu \nu}=\left(\begin{array}{c}
\vec{A}_{\mu \nu} \\
\vec{B}_{\mu \nu}
\end{array}\right)=\left(\begin{array}{c}
\hat{A}_{\mu \nu}+\vec{X}_{\mu \nu} \\
\hat{B}_{\mu \nu}+\vec{Y}_{\mu \nu}
\end{array}\right),
$$

we have

$$
\begin{gathered}
\vec{A}_{\mu \nu}=L_{\mu \nu} \hat{n}+\dot{\vec{X}}_{\mu \nu} \\
=L_{\mu \nu}^{1} \hat{n}_{1}+L_{\mu \nu}^{2} \hat{n}_{2}+L_{\mu \nu} \hat{n}, \\
\vec{B}_{\mu \nu}=\widetilde{L}_{\mu \nu} \hat{n}+\dot{\vec{Y}}_{\mu \nu} \\
=\widetilde{L}_{\mu \nu}^{1} \hat{n}_{1}+\widetilde{L}_{\mu \nu}^{2} \hat{n}_{2}+\widetilde{L}_{\mu \nu} \hat{n} .
\end{gathered}
$$

This completes the $A_{2}$ decomposition of the gravitational connection.

\section{B. $B_{2}$ (Light-like) Isometry}

This is when the isometry group is made of $\left(L_{1}+\right.$ $\left.K_{2}\right) / \sqrt{2}$ and $\left(L_{2}-K_{1}\right) / \sqrt{2}$. Let $\mathbf{p}$ and $\tilde{\mathbf{p}}$ be the two isometry vector fields which correspond to $\left(L_{1}+K_{2}\right) / \sqrt{2}$ and $\left(L_{2}-K_{1}\right) / \sqrt{2}$ which are dual to each other. In this case we can write

$$
\begin{gathered}
\mathbf{p}=f\left(\frac{\mathbf{l}_{1}+\mathbf{k}_{2}}{\sqrt{2}}\right)=\frac{f}{\sqrt{2}}\left(\begin{array}{c}
\hat{n}_{1} \\
\hat{n}_{2}
\end{array}\right), \\
\tilde{\mathbf{p}}=f\left(\frac{\mathbf{l}_{2}-\mathbf{k}_{1}}{\sqrt{2}}\right)=\frac{f}{\sqrt{2}}\left(\begin{array}{c}
\hat{n}_{2} \\
-\hat{n}_{1}
\end{array}\right) .
\end{gathered}
$$

But notice that the Casimir invariants $(\alpha, \beta)$ of the isometry vectors are given by $(0,0)$ independent of $f$. Moreover, here (unlike the $A_{2}$ case) the isometry condition does not restrict $f$ at all, because we have

$$
\partial_{\mu} \mathbf{p}^{2}=2 \mathbf{p} \cdot D_{\mu} \mathbf{p}=0
$$

independent of $f$. So the $B_{2}$ isometry vectors contain an arbitrary scalar function $f(x)$. 
Let us put $f=e^{\lambda}$ and express the $B_{2}$ isometry by

$$
\begin{gathered}
\mathbf{j}=\frac{e^{\lambda}}{\sqrt{2}}\left(\mathbf{l}_{1}+\mathbf{k}_{2}\right)=\frac{e^{\lambda}}{\sqrt{2}}\left(\begin{array}{c}
\hat{n}_{1} \\
\hat{n}_{2}
\end{array}\right), \\
\tilde{\mathbf{j}}=\frac{e^{\lambda}}{\sqrt{2}}\left(\mathbf{l}_{2}-\mathbf{k}_{1}\right)=\frac{e^{\lambda}}{\sqrt{2}}\left(\begin{array}{c}
\hat{n}_{2} \\
-\hat{n}_{1}
\end{array}\right), \\
D_{\mu} \mathbf{j}=0, \quad D_{\mu} \tilde{\mathbf{j}}=0,
\end{gathered}
$$

To find the restricted connection $\hat{\boldsymbol{\Gamma}}$ which satisfies the isometry condition we first introduce 4 more basis vectors in Lorentz group manifold which together with $\mathbf{j}$ and $\tilde{\mathbf{j}}$ form a complete basis

$$
\begin{gathered}
\mathbf{k}=\frac{e^{-\lambda}}{\sqrt{2}}\left(\mathbf{l}_{1}-\mathbf{k}_{2}\right)=\frac{e^{-\lambda}}{\sqrt{2}}\left(\begin{array}{c}
\hat{n}_{1} \\
-\hat{n}_{2}
\end{array}\right), \\
\tilde{\mathbf{k}}=-\frac{e^{-\lambda}}{\sqrt{2}}\left(\mathbf{l}_{2}+\mathbf{k}_{1}\right)=\frac{e^{-\lambda}}{\sqrt{2}}\left(\begin{array}{c}
-\hat{n}_{2} \\
-\hat{n}_{1}
\end{array}\right), \\
\mathbf{l}=-\mathbf{j} \times \tilde{\mathbf{k}}=-\tilde{\mathbf{j}} \times \mathbf{k}=\left(\begin{array}{c}
\hat{n}_{3} \\
0
\end{array}\right), \\
\tilde{\mathbf{l}}=\mathbf{j} \times \mathbf{k}=-\tilde{\mathbf{j}} \times \tilde{\mathbf{k}}=\left(\begin{array}{c}
0 \\
-\hat{n}_{3}
\end{array}\right) .
\end{gathered}
$$

Notice that 4 of them are null vectors,

$$
\mathbf{j}^{2}=\tilde{\mathbf{j}}^{2}=\mathbf{k}^{2}=\tilde{\mathbf{k}}^{2}=0,
$$

but we have

$$
\mathbf{j} \cdot \mathbf{k}=-\tilde{\mathbf{j}} \cdot \tilde{\mathbf{k}}=1, \quad \mathbf{l}^{2}=-\tilde{\mathbf{l}}^{2}=1
$$

All other scalar products of the basis vectors vanish. Moreover we have

$$
\begin{gathered}
\mathbf{j} \times \mathbf{l}=-\tilde{\mathbf{j}} \times \tilde{\mathbf{l}}=-\tilde{\mathbf{j}}, \quad \tilde{\mathbf{j}} \times \mathbf{l}=\mathbf{j} \times \tilde{\mathbf{l}}=\mathbf{j}, \\
\mathbf{k} \times \mathbf{l}=-\tilde{\mathbf{k}} \times \tilde{\mathbf{l}}=\tilde{\mathbf{k}}, \quad \tilde{\mathbf{k}} \times \mathbf{l}=\mathbf{k} \times \tilde{\mathbf{l}}=-\mathbf{k} .
\end{gathered}
$$

From this we find the following restricted connection for the $B_{2}$ isometry,

$$
\begin{gathered}
\hat{\boldsymbol{\Gamma}}_{\mu}=\Gamma_{\mu} \mathbf{j}-\widetilde{\Gamma}_{\mu} \tilde{\mathbf{j}}-\mathbf{k} \times \partial_{\mu} \mathbf{j} \\
=\Gamma_{\mu} \mathbf{j}-\widetilde{\Gamma}_{\mu} \tilde{\mathbf{j}}-\frac{1}{2}\left(\mathbf{k} \times \partial_{\mu} \mathbf{j}-\tilde{\mathbf{k}} \times \partial_{\mu}, \tilde{\mathbf{j}}\right) \\
\Gamma_{\mu}=\mathbf{k} \cdot \boldsymbol{\Gamma}_{\mu}, \quad \widetilde{\Gamma}_{\mu}=\tilde{\mathbf{k}} \cdot \boldsymbol{\Gamma}_{\mu} \\
\mathbf{k} \times \partial_{\mu} \mathbf{j}=-\tilde{\mathbf{k}} \times \partial_{\mu} \tilde{\mathbf{j}}
\end{gathered}
$$

where $\Gamma_{\mu}$ and $\widetilde{\Gamma}_{\mu}$ are two Abelian connections of $\mathbf{j}$ and $\tilde{\mathbf{j}}$ components which are not restricted by the isometry condition.

The restricted curvature tensor $\hat{\mathbf{R}}_{\mu \nu}$ is given by

$$
\begin{gathered}
\hat{\mathbf{R}}_{\mu \nu}=\partial_{\mu} \hat{\boldsymbol{\Gamma}}_{\nu}-\partial_{\nu} \hat{\boldsymbol{\Gamma}}_{\mu}+\hat{\boldsymbol{\Gamma}}_{\mu} \times \hat{\boldsymbol{\Gamma}}_{\nu} \\
=\left(\Gamma_{\mu \nu}+H_{\mu \nu}\right) \mathbf{j}-\left(\widetilde{\Gamma}_{\mu \nu}+\widetilde{H}_{\mu \nu}\right) \tilde{\mathbf{j}} \\
\Gamma_{\mu \nu}=\partial_{\mu} \Gamma_{\nu}-\partial_{\nu} \Gamma_{\mu}, \quad \widetilde{\Gamma}_{\mu \nu}=\partial_{\mu} \widetilde{\Gamma}_{\nu}-\partial_{\nu} \widetilde{\Gamma}_{\mu}, \\
H_{\mu \nu}=-\mathbf{k} \cdot\left(\partial_{\mu} \mathbf{j} \times \partial_{\nu} \mathbf{k}-\partial_{\nu} \mathbf{j} \times \partial_{\mu} \mathbf{k}\right), \\
\widetilde{H}_{\mu \nu}=-\tilde{\mathbf{k}} \cdot\left(\partial_{\mu} \mathbf{j} \times \partial_{\nu} \mathbf{k}-\partial_{\nu} \mathbf{j} \times \partial_{\mu} \mathbf{k}\right),
\end{gathered}
$$

so that

$$
\hat{R}_{\mu \nu}^{a b}=\left(\Gamma_{\mu \nu}+H_{\mu \nu}\right) j^{a b}-\left(\widetilde{\Gamma}_{\mu \nu}+\widetilde{H}_{\mu \nu}\right) \tilde{j}^{a b} .
$$

This should be contrasted with the restricted curvature tensor (76) of the $A_{2}$ isometry.

In 3-dimensional notation the isometry condition (101) is written as

$$
\begin{gathered}
\hat{\boldsymbol{\Gamma}}_{\mu}=\left(\begin{array}{c}
\hat{A}_{\mu} \\
\hat{B}_{\mu}
\end{array}\right), \\
\hat{D}_{\mu} \hat{n}_{1}=\hat{B}_{\mu} \times \hat{n}_{2}-\left(\partial_{\mu} \lambda\right) \hat{n}_{1}, \\
\hat{D}_{\mu} \hat{n}_{2}=-\hat{B}_{\mu} \times \hat{n}_{1}-\left(\partial_{\mu} \lambda\right) \hat{n}_{2} .
\end{gathered}
$$

From this we have

$$
\begin{gathered}
\hat{A}_{\mu}=A_{\mu}^{1} \hat{n}_{1}+A_{\mu}^{2} \hat{n}_{2}+\left(\hat{n}_{1} \cdot \partial_{\mu} \hat{n}_{2}\right) \hat{n}_{3} \\
=\left(\frac{e^{\lambda}}{\sqrt{2}} \Gamma_{\mu}+\frac{\hat{n}_{2} \cdot \partial_{\mu} \hat{n}_{3}}{2}\right) \hat{n}_{1}-\left(\frac{e^{\lambda}}{\sqrt{2}} \widetilde{\Gamma}_{\mu}+\frac{\hat{n}_{1} \cdot \partial_{\mu} \hat{n}_{3}}{2}\right) \hat{n}_{2} \\
+\left(\hat{n}_{1} \cdot \partial_{\mu} \hat{n}_{2}\right) \hat{n}_{3}, \\
\hat{B}_{\mu}=B_{\mu}^{1} \hat{n}_{1}+B_{\mu}^{2} \hat{n}_{2}-\left(\partial_{\mu} \lambda\right) \hat{n}_{3} \\
=\left(\frac{e^{\lambda}}{\sqrt{2}} \widetilde{\Gamma}_{\mu}-\frac{\hat{n}_{1} \cdot \partial_{\mu} \hat{n}_{3}}{2}\right) \hat{n}_{1}+\left(\frac{e^{\lambda}}{\sqrt{2}} \Gamma_{\mu}-\frac{\hat{n}_{2} \cdot \partial_{\mu} \hat{n}_{3}}{2}\right) \hat{n}_{2} \\
A_{\mu}^{1}=\frac{e^{\lambda}}{\sqrt{2}}\left(\Gamma_{\mu}-\tilde{C}_{\mu}^{1}\right), \quad \hat{n}_{\mu}^{2}=-\frac{e^{\lambda}}{\sqrt{2}}\left(\widetilde{\Gamma}_{\mu}-\widetilde{C}_{\mu}^{2}\right), \\
B_{\mu}^{1}=\frac{e^{\lambda}}{\sqrt{2}}\left(\widetilde{\Gamma}_{\mu}+\widetilde{C}_{\mu}^{2}\right), \quad B_{\mu}^{2}=\frac{e^{\lambda}}{\sqrt{2}}\left(\Gamma_{\mu}+\widetilde{C}_{\mu}^{1}\right), \\
\widetilde{C}_{\mu}^{1}=-\frac{e^{-\lambda}}{\sqrt{2}} \hat{n}_{2} \cdot \partial_{\mu} \hat{n}_{3}, \\
\widetilde{C}_{\mu}^{2}=-\frac{e^{-\lambda}}{\sqrt{2}} \hat{n}_{1} \cdot \partial_{\mu} \hat{n}_{3},
\end{gathered}
$$

so that

$$
\begin{gathered}
\hat{A}_{\mu}=-\hat{n}_{3} \times \hat{B}_{\mu}+\frac{1}{2} \epsilon_{i j k}\left(\hat{n}_{i} \times \hat{n}_{j}\right) \hat{n}_{k} \\
=B_{\mu}^{2} \hat{n}_{1}-B_{\mu}^{1} \hat{n}_{2}+\frac{1}{2} \epsilon_{i j k}\left(\hat{n}_{i} \times \hat{n}_{j}\right) \hat{n}_{k}, \\
\hat{B}_{\mu}=\hat{n}_{3} \times \hat{A}_{\mu}-\partial_{\mu} \hat{n}_{3}-\left(\partial_{\mu} \lambda\right) \hat{n}_{3} \\
=-A_{\mu}^{2} \hat{n}_{1}+A_{\mu}^{1} \hat{n}_{2}-\partial_{\mu} \hat{n}_{3}-\left(\partial_{\mu} \lambda\right) \hat{n}_{3} .
\end{gathered}
$$

Notice that both $\hat{A}_{\mu}$ and $\hat{B}_{\mu}$ have non-vanishing $\hat{n}_{3}$ components.

With

$$
\hat{\mathbf{R}}_{\mu \nu}=\left(\begin{array}{c}
\hat{A}_{\mu \nu} \\
\hat{B}_{\mu \nu}
\end{array}\right)
$$

we have

$$
\begin{aligned}
& \hat{A}_{\mu \nu}=\partial_{\mu} \hat{A}_{\nu}-\partial_{\nu} \hat{A}_{\mu}+\hat{A}_{\mu} \times \hat{A}_{\nu}-\hat{B}_{\mu} \times \hat{B}_{\nu} \\
& =\frac{e^{\lambda}}{\sqrt{2}}\left(\Gamma_{\mu \nu}+H_{\mu \nu}\right) \hat{n}_{1}-\frac{e^{\lambda}}{\sqrt{2}}\left(\widetilde{\Gamma}_{\mu \nu}+\widetilde{H}_{\mu \nu}\right) \hat{n}_{2}
\end{aligned}
$$




$$
\begin{gathered}
=A_{\mu \nu}^{1} \hat{n}_{1}+A_{\mu \nu}^{2} \hat{n}_{2}, \\
\hat{B}_{\mu \nu}=\partial_{\mu} \hat{B}_{\nu}-\partial_{\nu} \hat{B}_{\mu}+\hat{A}_{\mu} \times \hat{B}_{\nu}+\hat{B}_{\mu} \times \hat{A}_{\nu} \\
=\hat{D}_{\mu} \hat{B}_{\nu}-\hat{D}_{\nu} \hat{B}_{\mu} \\
=\frac{e^{\lambda}}{\sqrt{2}}\left(\widetilde{\Gamma}_{\mu \nu}+\widetilde{H}_{\mu \nu}\right) \hat{n}_{1}+\frac{e^{\lambda}}{\sqrt{2}}\left(\Gamma_{\mu \nu}+H_{\mu \nu}\right) \hat{n}_{2} \\
=B_{\mu \nu}^{1} \hat{n}_{1}+B_{\mu \nu}^{2} \hat{n}_{2},
\end{gathered}
$$

where

$$
\begin{gathered}
H_{\mu \nu}=\partial_{\mu} \widetilde{C}_{\nu}^{1}-\partial_{\nu} \widetilde{C}_{\nu}^{1}=\frac{e^{-\lambda}}{\sqrt{2}}\left(-\hat{n}_{1} \cdot\left(\partial_{\mu} \hat{n}_{1} \times \partial_{\nu} \hat{n}_{1}\right)\right. \\
\left.+\hat{n}_{2} \cdot\left(\partial_{\mu} \lambda \partial_{\nu} \hat{n}_{3}-\partial_{\nu} \lambda \partial_{\mu} \hat{n}_{3}\right)\right) \\
\widetilde{H}_{\mu \nu}=\partial_{\mu} \widetilde{C}_{\nu}^{2}-\partial_{\nu} \widetilde{C}_{\nu}^{2}=\frac{e^{-\lambda}}{\sqrt{2}}\left(\hat{n}_{2} \cdot\left(\partial_{\mu} \hat{n}_{2} \times \partial_{\nu} \hat{n}_{2}\right)\right. \\
\left.-\hat{n}_{3} \cdot\left(\partial_{\mu} \lambda \partial_{\nu} \hat{n}_{1}-\partial_{\nu} \lambda \partial_{\mu} \hat{n}_{1}\right)\right) \\
A_{\mu \nu}^{1}=B_{\mu \nu}^{2}=\frac{e^{\lambda}}{\sqrt{2}}\left(\partial_{\mu} K_{\nu}-\partial_{\nu} K_{\mu}\right) \\
A_{\mu \nu}^{2}=-B_{\mu \nu}^{1}=-\frac{e^{\lambda}}{\sqrt{2}}\left(\partial_{\mu} \widetilde{K}_{\nu}-\partial_{\nu} \widetilde{K}_{\mu}\right) \\
K_{\mu}=\Gamma_{\mu}+\widetilde{C}_{\mu}^{1}, \quad \widetilde{K}_{\mu}=\widetilde{\Gamma}_{\mu}+\widetilde{C}_{\mu}^{2}
\end{gathered}
$$

so that

$$
\hat{A}_{\mu \nu}=-\hat{n}_{3} \times \hat{B}_{\mu \nu}, \quad \hat{B}_{\mu \nu}=\hat{n}_{3} \times \hat{A}_{\mu \nu} .
$$

Notice that both $\hat{A}_{\mu \nu}$ and $\hat{B}_{\mu \nu}$ are orthogonal to $\hat{n}_{3}$, although $\hat{A}_{\mu}$ and $\hat{B}_{\mu}$ are not.

With this we obtain the full gauge potential of Lorentz group by adding the valence connection $\mathbf{Z}_{\mu}$,

$$
\begin{gathered}
\boldsymbol{\Gamma}_{\mu}=\hat{\boldsymbol{\Gamma}}_{\mu}+\mathbf{Z}_{\mu}, \\
\mathbf{k} \cdot \mathbf{Z}_{\mu}=\tilde{\mathbf{k}} \cdot \mathbf{Z}_{\mu}=0 .
\end{gathered}
$$

With

$$
\begin{gathered}
\mathbf{Z}_{\mu}=J_{\mu} \mathbf{k}-\widetilde{J}_{\mu} \tilde{\mathbf{k}}+L_{\mu} \mathbf{l}-\widetilde{L}_{\mu} \tilde{\mathbf{l}} \\
J_{\mu}=\mathbf{j} \cdot \mathbf{Z}_{\mu}, \quad \widetilde{J}_{\mu}=\tilde{\mathbf{j}} \cdot \mathbf{Z}_{\mu} \\
L_{\mu}=\mathbf{l} \cdot \mathbf{Z}_{\mu}, \quad \widetilde{L}_{\mu}=\tilde{\mathbf{l}} \cdot \mathbf{Z}_{\mu}
\end{gathered}
$$

we have

$$
\begin{gathered}
\dot{\mathbf{Z}}_{\mu \nu}=\hat{D}_{\mu} \mathbf{Z}_{\nu}-\hat{D}_{\nu} \mathbf{Z}_{\mu} \\
=U_{\mu \nu} \mathbf{j}-\widetilde{U}_{\mu \nu} \tilde{\mathbf{j}}+\left(\partial_{\mu} J_{\nu}-\partial_{\nu} J_{\mu}\right) \mathbf{k}-\left(\partial_{\mu} \widetilde{J}_{\nu}-\partial_{\nu} \widetilde{J}_{\mu}\right) \tilde{\mathbf{k}} \\
+\left(\mathcal{D}_{\mu} L_{\nu}-\mathcal{D}_{\nu} L_{\mu}\right) \mathbf{l}-\left(\mathcal{D}_{\mu} \widetilde{L}_{\nu}-\mathcal{D}_{\nu} \widetilde{L}_{\mu}\right) \tilde{\mathbf{l}} \\
U_{\mu \nu}=\widetilde{K}_{\mu} L_{\nu}-K_{\mu} \widetilde{L}_{\nu}-\widetilde{K}_{\nu} L_{\mu}+K_{\nu} \widetilde{L}_{\mu}, \\
\widetilde{U}_{\mu \nu}=K_{\mu} L_{\nu}+\widetilde{K}_{\mu} \widetilde{L}_{\nu}-K_{\nu} L_{\mu}-\widetilde{K}_{\nu} \widetilde{L}_{\mu}, \\
\mathcal{D}_{\mu} L_{\nu}=\partial_{\mu} L_{\nu}+K_{\mu} \widetilde{J}_{\nu}-\widetilde{K}_{\mu} J_{\nu}, \\
\mathcal{D}_{\mu} \widetilde{L}_{\nu}=\partial_{\mu} \widetilde{L}_{\nu}-K_{\mu} J_{\nu}-\widetilde{K}_{\mu} \widetilde{J}_{\nu} \\
\mathbf{Z}_{\mu \nu}^{\prime}=\mathbf{Z}_{\mu} \times \mathbf{Z}_{\nu}=V_{\mu \nu} \mathbf{k}+\widetilde{V}_{\mu \nu} \tilde{\mathbf{k}} \\
V_{\mu \nu}=J_{\mu} \widetilde{L}_{\nu}+\widetilde{J}_{\mu} L_{\nu}-J_{\nu} \widetilde{L}_{\mu}-\widetilde{J}_{\nu} L_{\mu}, \\
\widetilde{V}_{\mu \nu}=\widetilde{J}_{\mu} \widetilde{L}_{\nu}-J_{\mu} L_{\nu}+J_{\nu} L_{\mu}-\widetilde{J}_{\nu} \widetilde{L}_{\mu},
\end{gathered}
$$

so that

$$
\begin{gathered}
\mathbf{Z}_{\mu \nu}=\dot{\mathbf{Z}}_{\mu \nu}+\mathbf{Z}_{\mu \nu}^{\prime}=U_{\mu \nu} \mathbf{j}-\widetilde{U}_{\mu \nu} \tilde{\mathbf{j}} \\
+\left(\mathcal{D}_{\mu} J_{\nu}-\mathcal{D}_{\nu} J_{\mu}\right) \mathbf{k}-\left(\mathcal{D}_{\mu} \widetilde{J}_{\nu}-\mathcal{D}_{\nu} \widetilde{J}_{\mu}\right) \tilde{\mathbf{k}} \\
+\left(\mathcal{D}_{\mu} L_{\nu}-\mathcal{D}_{\nu} L_{\mu}\right) \mathbf{l}-\left(\mathcal{D}_{\mu} \widetilde{L}_{\nu}-\mathcal{D}_{\nu} \widetilde{L}_{\mu}\right) \tilde{\mathbf{l}}, \\
\mathcal{D}_{\mu} J_{\nu}=\partial_{\mu} J_{\nu}-\widetilde{L}_{\mu} J_{\nu}-L_{\mu} \widetilde{J}_{\nu}, \\
\mathcal{D}_{\mu} \widetilde{J}_{\nu}=\partial_{\mu} \widetilde{J}_{\nu}-\widetilde{L}_{\mu} \widetilde{J}_{\nu}-L_{\mu} J_{\nu} .
\end{gathered}
$$

Notice that in this case the kinetic part $\dot{\mathbf{Z}}_{\mu \nu}$ contains all six components, but the potential part $\mathbf{Z}_{\mu \nu}^{\prime}$ has only $\mathbf{k}$ and $\tilde{\mathbf{k}}$ components. With this we have the full curvature tensor

$$
\begin{gathered}
\mathbf{R}_{\mu \nu}=\hat{\mathbf{R}}_{\mu \nu}+\dot{\mathbf{Z}}_{\mu \nu}+\mathbf{Z}_{\mu \nu}^{\prime} \\
=\left(\Gamma_{\mu \nu}+H_{\mu \nu}+U_{\mu \nu}\right) \mathbf{j}-\left(\widetilde{\Gamma}_{\mu \nu}+\widetilde{H}_{\mu \nu}+\widetilde{U}_{\mu \nu}\right) \tilde{\mathbf{j}} \\
+\left(\mathcal{D}_{\mu} J_{\nu}-\mathcal{D}_{\nu} J_{\mu}\right) \mathbf{k}-\left(\mathcal{D}_{\mu} \widetilde{J}_{\nu}-\mathcal{D}_{\nu} \widetilde{J}_{\mu}\right) \tilde{\mathbf{k}} \\
+\left(\mathcal{D}_{\mu} L_{\nu}-\mathcal{D}_{\nu} L_{\mu}\right) \mathbf{l}-\left(\mathcal{D}_{\mu} \widetilde{L}_{\nu}-\mathcal{D}_{\nu} \widetilde{L}_{\mu}\right) \tilde{\mathbf{l}} \\
=\left(\mathcal{D}_{\mu} K_{\nu}-\mathcal{D}_{\nu} K_{\mu}\right) \mathbf{j}-\left(\mathcal{D}_{\mu} \widetilde{K}_{\nu}-\mathcal{D}_{\nu} \widetilde{K}_{\mu}\right) \tilde{\mathbf{j}} \\
+\left(\mathcal{D}_{\mu} J_{\nu}-\mathcal{D}_{\nu} J_{\mu}\right) \mathbf{k}-\left(\mathcal{D}_{\mu} \widetilde{J}_{\nu}-\mathcal{D}_{\nu} \widetilde{J}_{\mu}\right) \tilde{\mathbf{k}} \\
+\left(\mathcal{D}_{\mu} L_{\nu}-\mathcal{D}_{\nu} L_{\mu}\right) \mathbf{l}-\left(\mathcal{D}_{\mu} \widetilde{L}_{\nu}-\mathcal{D}_{\nu} \widetilde{L}_{\mu}\right) \tilde{\mathbf{l}} \\
=K_{\mu \nu} \mathbf{j}-\widetilde{K}_{\mu \nu} \tilde{\mathbf{j}}+J_{\mu \nu} \mathbf{k}-\widetilde{J}_{\mu \nu} \tilde{\mathbf{k}} \\
+L_{\mu \nu} \mathbf{l}-\widetilde{L}_{\mu \nu} \tilde{\mathbf{l}} \\
\mathcal{D}_{\mu} K_{\nu}=\partial_{\mu} K_{\nu}+\widetilde{L}_{\mu} K_{\nu}-L_{\mu} \widetilde{K}_{\nu} \\
\mathcal{D}_{\mu} \widetilde{K}_{\nu}=\partial_{\mu} \widetilde{K}_{\nu}-\widetilde{L}_{\mu} \widetilde{K}_{\nu}-L_{\mu} K_{\nu} \\
K_{\mu \nu}=\Gamma_{\mu \nu}+H_{\mu \nu}+U_{\mu \nu}=\mathcal{D}_{\mu} K_{\nu}-\mathcal{D}_{\nu} K_{\mu} \\
\widetilde{K}_{\mu \nu}=\widetilde{\Gamma}_{\mu \nu}+\widetilde{H}_{\mu \nu}+\widetilde{U}_{\mu \nu}=\mathcal{D}_{\mu} \widetilde{K}_{\nu}-\mathcal{D}_{\nu} \widetilde{K}_{\mu} \\
J_{\mu \nu}=\mathcal{D}_{\mu} J_{\nu}-\mathcal{D}_{\nu} J_{\mu}, \quad \widetilde{J}_{\mu \nu}=\mathcal{D}_{\mu} \widetilde{J}_{\nu}-\mathcal{D}_{\nu} \widetilde{J}_{\mu} \\
L_{\mu \nu}=\mathcal{D}_{\mu} L_{\nu}-\mathcal{D}_{\nu} L_{\mu} \\
\widetilde{L}_{\mu \nu}=\mathcal{D}_{\mu} \widetilde{L}_{\nu}-\mathcal{D}_{\nu} \widetilde{L}_{\mu}
\end{gathered}
$$

or equivalently

$$
\begin{gathered}
R_{\mu \nu}^{a b}=\mathbf{R}_{\mu \nu} \cdot \mathbf{I}^{a b} \\
=K_{\mu \nu} j^{a b}-\widetilde{K}_{\mu \nu} \tilde{j}^{a b}+J_{\mu \nu} k^{a b}-\widetilde{J}_{\mu \nu} \tilde{k}^{a b} \\
+L_{\mu \nu} l^{a b}-\widetilde{L}_{\mu \nu} \tilde{l}^{a b}
\end{gathered}
$$

This is the $B_{2}$ decomposition of the curvature tensor.

With complex notation

$$
\begin{gathered}
\mathbf{k}_{ \pm}=\frac{1}{\sqrt{2}}(\mathbf{k} \pm i \mathbf{l}), \quad \tilde{\mathbf{k}}_{ \pm}=\frac{1}{\sqrt{2}}(\tilde{\mathbf{k}} \pm i \tilde{\mathbf{l}}), \\
Z_{\mu}=\frac{1}{\sqrt{2}}\left(J_{\mu}+i L_{\mu}\right), \quad \widetilde{Z}_{\mu}=\frac{1}{\sqrt{2}}\left(\widetilde{J}_{\mu}+i \widetilde{L}_{\mu}\right), \\
Z_{\mu}^{\prime}=\frac{1}{\sqrt{2}}\left(K_{\mu}+i L_{\mu}\right)=Z_{\mu}-\frac{1}{\sqrt{2}} B_{\mu}^{-}, \\
\widetilde{Z}_{\mu}^{\prime}=\frac{1}{\sqrt{2}}\left(\widetilde{K}_{\mu}+i \widetilde{L}_{\mu}\right)=\widetilde{Z}_{\mu}-\frac{1}{\sqrt{2}} \widetilde{B}_{\mu}^{-}, \\
B_{\mu}^{ \pm}=J_{\mu} \pm K_{\mu}, \quad \widetilde{B}_{\mu}^{ \pm}=\widetilde{J}_{\mu} \pm \widetilde{K}_{\mu},
\end{gathered}
$$


we obtain

$$
\begin{gathered}
\mathbf{Z}_{\mu \nu}=-i\left(Z_{\mu}^{*} Z_{\nu}^{\prime}-Z_{\nu}^{*} Z_{\mu}^{\prime}-\widetilde{Z}_{\mu}^{* *} \widetilde{Z}_{\nu}^{\prime}+\widetilde{Z}_{\nu}^{\prime *} \widetilde{Z}_{\mu}^{\prime}\right) \mathbf{j} \\
+i\left(Z_{\mu}^{\prime *} \widetilde{Z}_{\nu}^{\prime}-Z_{\nu}^{\prime *} \widetilde{Z}_{\mu}^{\prime}-\widetilde{Z}_{\mu}^{* *} Z_{\nu}^{\prime}+\widetilde{Z}_{\nu}^{\prime *} Z_{\mu}^{\prime}\right) \tilde{\mathbf{j}} \\
+\left(\mathcal{D}_{\mu} Z_{\nu}-\mathcal{D}_{\nu} Z_{\mu}\right)^{*} \mathbf{k}_{+}-\left(\mathcal{D}_{\mu} \widetilde{Z}_{\nu}-\mathcal{D}_{\nu} \widetilde{Z}_{\mu}\right)^{*} \tilde{\mathbf{k}}_{+} \\
+\left(\mathcal{D}_{\mu} Z_{\nu}-\mathcal{D}_{\nu} Z_{\mu}\right) \mathbf{k}_{-}-\left(\mathcal{D}_{\mu} \widetilde{Z}_{\nu}-\mathcal{D}_{\nu} \widetilde{Z}_{\mu}\right) \tilde{\mathbf{k}}_{-} \\
\mathcal{D}_{\mu} Z_{\nu}=\left(\partial_{\mu}-i \widetilde{B}_{\mu}^{+}\right) Z_{\nu}-i B_{\mu}^{+} \widetilde{Z}_{\nu}^{*} \\
+i \widetilde{B}_{\mu}^{-} Z_{\nu}^{*}+i B_{\mu}^{-} \widetilde{Z}_{\nu}
\end{gathered}
$$

With this we have

$$
\begin{gathered}
\mathbf{R}_{\mu \nu}=\left(\mathcal{D}_{\mu} K_{\nu}-\mathcal{D}_{\nu} K_{\mu}\right) \mathbf{j}-\left(\mathcal{D}_{\mu} \widetilde{K}_{\nu}-\mathcal{D}_{\nu} \widetilde{K}_{\mu}\right) \tilde{\mathbf{j}} \\
+\left(\mathcal{D}_{\mu} Z_{\nu}-\mathcal{D}_{\nu} Z_{\mu}\right)^{*} \mathbf{k}_{+}-\left(\mathcal{D}_{\mu} \widetilde{Z}_{\nu}-\mathcal{D}_{\nu} \widetilde{Z}_{\mu}\right)^{*} \tilde{\mathbf{k}}_{+} \\
+\left(\mathcal{D}_{\mu} Z_{\nu}-\mathcal{D}_{\nu} Z_{\mu}\right) \mathbf{k}_{-} \\
-\left(\mathcal{D}_{\mu} \widetilde{Z}_{\nu}-\mathcal{D}_{\nu} \widetilde{Z}_{\mu}\right) \tilde{\mathbf{k}}_{-}
\end{gathered}
$$

or

$$
\begin{gathered}
R_{\mu \nu}^{a b}=\left(\mathcal{D}_{\mu} K_{\nu}-\mathcal{D}_{\nu} K_{\mu}\right) j^{a b}-\left(\mathcal{D}_{\mu} \widetilde{K}_{\nu}-\mathcal{D}_{\nu} \widetilde{K}_{\mu}\right) \tilde{j}^{a b} \\
+\left(\mathcal{D}_{\mu} Z_{\nu}-\mathcal{D}_{\nu} Z_{\mu}\right)^{*} k_{+}^{a b}-\left(\mathcal{D}_{\mu} \widetilde{Z}_{\nu}-\mathcal{D}_{\nu} \widetilde{Z}_{\mu}\right)^{*} \tilde{k}_{+}^{a b} \\
+\left(\mathcal{D}_{\mu} Z_{\nu}-\mathcal{D}_{\nu} Z_{\mu}\right) k_{-}^{a b} \\
-\left(\mathcal{D}_{\mu} \widetilde{Z}_{\nu}-\mathcal{D}_{\nu} \widetilde{Z}_{\mu}\right) \tilde{k}_{-}^{a b}
\end{gathered}
$$

This should be compared with the $a_{2}$ result (92) or (93).

In 3-dimensional notation, we have

$$
\begin{gathered}
\mathbf{Z}_{\mu}=\left(\begin{array}{c}
\vec{X}_{\mu} \\
\vec{Y}_{\mu}
\end{array}\right), \\
\vec{X}_{\mu}=\frac{e^{-\lambda}}{\sqrt{2}}\left(J_{\mu} \hat{n}_{1}+\widetilde{J}_{\mu} \hat{n}_{2}+L_{\mu} \hat{n}_{3}\right), \\
\vec{Y}_{\mu}=\frac{e^{-\lambda}}{\sqrt{2}}\left(\widetilde{J}_{\mu} \hat{n}_{1}-J_{\mu} \hat{n}_{2}+\widetilde{L}_{\mu} \hat{n}_{3}\right),
\end{gathered}
$$

so that

$$
\begin{gathered}
\hat{n}_{1} \cdot \vec{X}_{\mu}+\hat{n}_{2} \cdot \vec{Y}_{\mu}=0, \\
\hat{n}_{2} \cdot \vec{X}_{\mu}-\hat{n}_{1} \cdot \vec{Y}_{\mu}=0, \\
\hat{n} \times \vec{Y}_{\mu}=-\hat{n} \times\left(\hat{n} \times \vec{X}_{\mu}\right) .
\end{gathered}
$$

Moreover, with

$$
\mathbf{Z}_{\mu \nu}=\left(\begin{array}{c}
\vec{X}_{\mu \nu} \\
\vec{Y}_{\mu \nu}
\end{array}\right)=\left(\begin{array}{c}
\dot{\vec{X}}_{\mu \nu}+\vec{X}_{\mu \nu}^{\prime} \\
\dot{\vec{Y}}_{\mu \nu}+\vec{Y}_{\mu \nu}^{\prime}
\end{array}\right),
$$

we have

$$
\begin{gathered}
\dot{\vec{X}}_{\mu \nu}=\left\{\frac{e^{\lambda}}{\sqrt{2}} U_{\mu \nu}+\frac{e^{-\lambda}}{\sqrt{2}}\left(\partial_{\mu} J_{\nu}-\partial_{\nu} J_{\mu}\right)\right\} \hat{n}_{1} \\
-\left\{\frac{e^{\lambda}}{\sqrt{2}} \widetilde{U}_{\mu \nu}-\frac{e^{-\lambda}}{\sqrt{2}}\left(\partial_{\mu} \widetilde{J}_{\nu}+\partial_{\nu} \widetilde{J}_{\mu}\right)\right\} \hat{n}_{2}+L_{\mu \nu} \hat{n}_{3}, \\
\dot{\vec{Y}}_{\mu \nu}=\left\{\frac{e^{\lambda}}{\sqrt{2}} \widetilde{U}_{\mu \nu}+\frac{e^{-\lambda}}{\sqrt{2}}\left(\partial_{\mu} \widetilde{J}_{\nu}-\partial_{\nu} \widetilde{J}_{\mu}\right)\right\} \hat{n}_{1}
\end{gathered}
$$

$$
\begin{gathered}
+\left\{\frac{e^{\lambda}}{\sqrt{2}} U_{\mu \nu}-\frac{e^{-\lambda}}{\sqrt{2}}\left(\partial_{\mu} J_{\nu}-\partial_{\nu} J_{\mu}\right)\right\} \hat{n}_{2}+\widetilde{L}_{\mu \nu} \hat{n}_{3}, \\
\vec{X}_{\mu \nu}^{\prime}=\frac{e^{-\lambda}}{\sqrt{2}}\left(V_{\mu \nu} \hat{n}_{1}+\widetilde{V}_{\mu \nu} \hat{n}_{2}\right), \\
\vec{Y}_{\mu \nu}^{\prime}=\frac{e^{-\lambda}}{\sqrt{2}}\left(\widetilde{V}_{\mu \nu} \hat{n}_{1}-V_{\mu \nu} \hat{n}_{2}\right),
\end{gathered}
$$

so that

$$
\begin{gathered}
\vec{X}_{\mu \nu}=\left(\frac{e^{\lambda}}{\sqrt{2}} U_{\mu \nu}+\frac{e^{-\lambda}}{\sqrt{2}} J_{\mu \nu}\right) \hat{n}_{1} \\
-\left(\frac{e^{\lambda}}{\sqrt{2}} \widetilde{U}_{\mu \nu}-\frac{e^{-\lambda}}{\sqrt{2}} \widetilde{J}_{\mu \nu}\right) \hat{n}_{2}+L_{\mu \nu} \hat{n}_{3}, \\
\vec{Y}_{\mu \nu}=\left(\frac{e^{\lambda}}{\sqrt{2}} \widetilde{U}_{\mu \nu}+\frac{e^{-\lambda}}{\sqrt{2}} \widetilde{J}_{\mu \nu}\right) \hat{n}_{1} \\
+\left(\frac{e^{\lambda}}{\sqrt{2}} U_{\mu \nu}-\frac{e^{-\lambda}}{\sqrt{2}} J_{\mu \nu}\right) \hat{n}_{2}+\widetilde{L}_{\mu \nu} \hat{n}_{3} .
\end{gathered}
$$

Finally with

$$
\mathbf{R}_{\mu \nu}=\left(\begin{array}{c}
\vec{A}_{\mu \nu} \\
\vec{B}_{\mu \nu}
\end{array}\right)=\left(\begin{array}{c}
\hat{A}_{\mu \nu}+\vec{X}_{\mu \nu} \\
\hat{B}_{\mu \nu}+\vec{Y}_{\mu \nu}
\end{array}\right),
$$

we have

$$
\begin{gathered}
\vec{A}_{\mu \nu}=\left(\frac{e^{\lambda}}{\sqrt{2}} K_{\mu \nu}+\frac{e^{-\lambda}}{\sqrt{2}} J_{\mu \nu}\right) \hat{n}_{1} \\
-\left(\frac{e^{\lambda}}{\sqrt{2}} \widetilde{K}_{\mu \nu}-\frac{e^{-\lambda}}{\sqrt{2}} \widetilde{J}_{\mu \nu}\right) \hat{n}_{2}+L_{\mu \nu} \hat{n}_{3} \\
\vec{B}_{\mu \nu}=\left(\frac{e^{\lambda}}{\sqrt{2}} \widetilde{K}_{\mu \nu}+\frac{e^{-\lambda}}{\sqrt{2}} \widetilde{J}_{\mu \nu}\right) \hat{n}_{1} \\
+\left(\frac{e^{\lambda}}{\sqrt{2}} K_{\mu \nu}-\frac{e^{-\lambda}}{\sqrt{2}} J_{\mu \nu}\right) \hat{n}_{2}+\widetilde{L}_{\mu \nu} \hat{n}_{3} .
\end{gathered}
$$

This completes the $B_{2}$ decomposition of the gravitational connection.

The above result tells that there exist two different Abelian decompositions of the gravitational connection and the curvature tensor which decompose them into the restricted part and the valence part. This allows us to decompose the Einstein's theory in terms of the restricted part and the valence part.

\section{DECOMPOSITION OF EINSTEIN'S THEORY}

Now we are ready to discuss the decomposition of Einstein's theory. Since the Einstein-Hilbert action is described by the metric we have to express the above decomposition of the gravitational connection in terms of the metric. To do this we use the first order formalism of Einstein theory. In the absence of the matter field, 
the Einstein-Hilbert action in the first order formalism is given by

$$
\begin{gathered}
S\left[e_{a}^{\mu}, \boldsymbol{\Gamma}_{\mu}\right]=\frac{1}{16 \pi G_{N}} \int\left(e e_{a}^{\mu} e_{b}^{\nu} \mathbf{I}^{a b} \cdot \mathbf{R}_{\mu \nu}\right) d^{4} x \\
=\frac{1}{16 \pi G_{N}} \int\left(\mathbf{g}_{\mu \nu} \cdot \mathbf{R}^{\mu \nu}\right) d^{4} x, \\
\mathbf{g}_{\mu \nu}=e e_{\mu}^{a} e_{\nu}^{b} \mathbf{I}_{a b}, \quad g_{\mu \nu}^{a b}=e\left(e_{\mu}^{a} e_{\nu}^{b}-e_{\nu}^{b} e_{\mu}^{a}\right), \\
e=\operatorname{Det}\left(e_{\mu a}\right) .
\end{gathered}
$$

Notice that here we have introduced the Lorentz covariant four index metric tensor $\mathbf{g}_{\mu \nu}$ (which should not be confused with the two index space-time metric $g_{\mu \nu}$ ) which forms an adjoint representation of Lorentz group. From (132) we have the following equation of motion

$$
\begin{gathered}
\delta e_{\mu a} ; \quad \mathbf{g}_{\mu \nu} \cdot \mathbf{R}^{\nu \rho} e_{\rho a}=R_{\mu a}=0 \\
\delta \boldsymbol{\Gamma}_{\mu} ; \quad D_{\mu} \mathbf{g}^{\mu \nu}=\left(\partial_{\mu}+\boldsymbol{\Gamma}_{\mu} \times\right) \mathbf{g}^{\mu \nu}=0,
\end{gathered}
$$

where $R_{\mu a}=e^{\nu b} R_{\mu \nu a b}$ is the Ricci tensor. Clearly the first equation is nothing but the Einstein's equation in the absence of matter field. But the Ricci tensor is written in terms of the gauge potential, not the metric. To understand the meaning of the second equation, notice that the second equation tells that $\mathbf{g}_{\mu \nu}$ is invariant under the parallel transport along the $\partial_{\mu}$-direction defined by the gauge potential $\boldsymbol{\Gamma}_{\mu}$. This, of course, puts a strong constraint on the gauge potential. In fact without much difficulty one can show that the second equation uniquely determines $\boldsymbol{\Gamma}_{\mu}$,

$$
\begin{gathered}
\boldsymbol{\Gamma}_{\mu} \cdot \mathbf{I}^{a b}=\Gamma_{\mu}^{a b} \\
=\frac{1}{2}\left(e^{a \nu} e_{c \mu} \partial^{b} e^{c}{ }_{\nu}+e^{a \nu} \partial_{\mu} e^{b}{ }_{\nu}+\partial^{b} e^{a}{ }_{\mu}\right. \\
\left.-e^{b \nu} e_{c \mu} \partial^{a} e^{c}{ }_{\nu}-e^{b \nu} \partial_{\mu} e^{a}{ }_{\nu}-\partial^{a} e^{b}{ }_{\mu}\right) .
\end{gathered}
$$

This, of course, is the well known equation of the spin connection $\omega_{\mu}^{a b}$, which confirms that the gauge potential $\boldsymbol{\Gamma}_{\mu}$ of Lorentz group becomes the spin connection. (Here $\Gamma_{\mu}^{a b}$ becomes the torsion-free spin connection, but notice that in general it can have torsion when a spinor source is present). So, the equation $D_{\mu} \mathbf{g}^{\mu \nu}=0$ becomes identical to the metric-compatibility condition of the connection

$$
\begin{gathered}
\nabla_{\alpha} g_{\mu \nu}=\partial_{\alpha} g_{\mu \nu}-\Gamma_{\alpha \mu}{ }^{\rho} g_{\rho \nu}-\Gamma_{\alpha \nu}{ }^{\rho} g_{\mu \rho}=0 \\
g_{\mu \nu}=\eta_{a b} e_{\mu}^{a} e_{\nu}^{b}
\end{gathered}
$$

which requires the space-time metric $g_{\mu \nu}$ to be invariant under the parallel transport defined by the connection. So in this formalism $D_{\mu} \mathbf{g}^{\mu \nu}=0$ plays the role of $\nabla_{\alpha} g_{\mu \nu}=$ 0 . This confirms that the above equation (133) describes the Einstein's general relativity.

With this preliminary, we discuss the decomposition of Einstein's theory with the $A_{2}$ isometry (the spacelike isometry) first. For this we introduce two projection operators which project out the isometry components,

$$
\boldsymbol{\Sigma}_{a b}=l_{a b} \mathbf{l}-\tilde{l}_{a b} \tilde{\mathbf{l}}
$$

$$
\begin{gathered}
\mathbf{\Pi}_{a b}=\mathbf{I}_{a b}-\boldsymbol{\Sigma}_{a b}=l_{a b}^{1} \mathbf{l}_{1}-\tilde{l}_{a b}^{1} \tilde{\mathbf{l}}_{1}+l_{a b}^{2} \mathbf{l}_{2}-\tilde{l}_{a b}^{2} \tilde{\mathbf{l}}_{2} \\
\Sigma_{a b}{ }^{c d}=l_{a b} l^{c d}-\tilde{l}_{a b} \tilde{l}^{c d} \\
\Pi_{a b}{ }^{c d}=I_{a b}{ }^{c d}-\Sigma_{a b}{ }^{c d} \\
\mathbf{Z}_{\mu} \cdot \boldsymbol{\Sigma}_{a b}=0, \quad \mathbf{Z}_{\mu} \cdot \mathbf{\Pi}_{a b}=Z_{\mu}{ }^{a b}
\end{gathered}
$$

Clearly $\boldsymbol{\Sigma}_{a b}$ and $\boldsymbol{\Pi}_{a b}$ become projection operators in the sense that

$$
\begin{gathered}
\boldsymbol{\Sigma}_{a b} \cdot \boldsymbol{\Sigma}^{c d}=\frac{1}{2} \Sigma_{a b}{ }^{m n} \Sigma_{m n}^{c d}=\Sigma_{a b}^{c d} \\
\boldsymbol{\Pi}_{a b} \cdot \boldsymbol{\Pi}^{c d}=\frac{1}{2} \Pi_{a b}{ }^{m n} \Pi_{m n}^{c d}=\Pi_{a b}^{c d} \\
\boldsymbol{\Sigma}_{a b} \cdot \boldsymbol{\Pi}^{c d}=0 .
\end{gathered}
$$

Now we can express the Einstein-Hilbert action as

$$
\begin{gathered}
S\left[e_{a}^{\mu}, \Gamma_{\mu}, \widetilde{\Gamma}_{\mu}, \mathbf{Z}_{\mu}\right]=\frac{1}{16 \pi G_{N}} \int\left\{\mathbf{g}_{\mu \nu} \cdot \mathbf{R}^{\mu \nu}\right. \\
\left.+\lambda\left(\mathbf{l}^{2}-1\right)+\tilde{\lambda}(\mathbf{l} \cdot \tilde{\mathbf{l}})+\lambda_{\mu}\left(\mathbf{l} \cdot \mathbf{Z}^{\mu}\right)+\tilde{\lambda}_{\mu}\left(\tilde{\mathbf{l}} \cdot \mathbf{Z}^{\mu}\right)\right\} d^{4} x \\
\mathbf{R}_{\mu \nu}=\hat{\mathbf{R}}_{\mu \nu}+\left(\hat{D}_{\mu} \mathbf{Z}_{\nu}-\hat{D}_{\nu} \mathbf{Z}_{\mu}\right)+\mathbf{Z}_{\mu} \times \mathbf{Z}_{\nu} \\
=\left(\mathcal{D}_{\mu} L_{\nu}-\mathcal{D}_{\nu} L_{\mu}\right) \mathbf{l}-\left(\mathcal{D}_{\mu} \widetilde{L}_{\nu}-\mathcal{D}_{\nu} \widetilde{L}_{\mu}\right) \tilde{\mathbf{l}} \\
+\left(\hat{D}_{\mu} \mathbf{Z}_{\nu}-\hat{D}_{\nu} \mathbf{Z}_{\mu}\right)
\end{gathered}
$$

where $\lambda^{\prime} s$ are the Lagrange multipliers. From this we get the following equations of motion

$$
\begin{gathered}
\delta e_{\mu c} ; \quad\left(e e_{\mu}^{a} e_{\nu}^{b}\right)\left[\left(\mathcal{D}^{\nu} L^{\rho}-\mathcal{D}^{\rho} L^{\nu}\right) l_{a b}\right. \\
\left.-\left(\mathcal{D}^{\nu} \widetilde{L}^{\rho}-\mathcal{D}^{\rho} \widetilde{L}^{\nu}\right) \tilde{l}_{a b}+\left(\hat{D}^{\nu} \mathbf{Z}^{\rho}-\hat{D}^{\rho} \mathbf{Z}^{\nu}\right) \cdot \mathbf{\Pi}_{a b}\right] e_{\rho c} \\
=0 \\
\delta \Gamma_{\nu} ; \quad \partial_{\mu}\left(e e_{a}^{\mu} e_{b}^{\nu} l^{a b}\right)+\mathbf{l} \cdot\left(\mathbf{Z}_{\mu} \times \mathbf{g}^{\mu \nu}\right)=0 \\
\delta \widetilde{\Gamma}_{\nu} ; \quad \partial_{\mu}\left(e e_{a}^{\mu} e_{b}^{\nu} \tilde{l}^{a b}\right)+\tilde{\mathbf{l}} \cdot\left(\mathbf{Z}_{\mu} \times \mathbf{g}^{\mu \nu}\right)=0 \\
\delta \mathbf{Z}_{\nu} ; \quad \hat{D}_{\mu}\left(e e_{a}^{\mu} e_{b}^{\nu} \mathbf{\Pi}^{a b}\right)+\left(e e_{a}^{\mu} e_{b}^{\nu}\right)\left[\left(\mathbf{Z}_{\mu} \times \mathbf{l}\right) l^{a b}\right. \\
\left.\left.-\mathbf{Z}_{\mu} \times \tilde{\mathbf{l}}\right) \tilde{l}^{a b}\right]=0 .
\end{gathered}
$$

Notice that, using the fact that $\hat{D}_{\mu} \mathbf{l}=\hat{D}_{\mu} \tilde{\mathbf{l}}=0$, we can combine the last three equations into a single equation,

$$
D_{\mu} \mathbf{g}^{\mu \nu}=0
$$

But this is precisely the second equation of (133), which confirms that (139) is equivalent to (133).

To clarify the meaning of the above equation we define the restricted metric $\hat{\mathbf{g}}_{\mu \nu}$ decomposing $\mathbf{g}_{\mu \nu}$

$$
\begin{gathered}
\mathbf{g}_{\mu \nu}=\hat{\mathbf{g}}_{\mu \nu}+\mathbf{G}_{\mu \nu}, \\
\hat{\mathbf{g}}_{\mu \nu}=e e_{\mu}^{a} e_{\nu}^{b} \mathbf{\Sigma}_{a b}=G_{\mu \nu} \mathbf{l}-\widetilde{G}_{\mu \nu} \tilde{\mathbf{l}}, \\
\mathbf{G}_{\mu \nu}=e e_{\mu}^{a} e_{\nu}^{b} \mathbf{\Pi}_{a b}=G_{\mu \nu}^{1} \mathbf{l}_{1}-\widetilde{G}_{\mu \nu}^{1} \tilde{\mathbf{l}}_{1} \\
+G_{\mu \nu}^{2} \mathbf{l}_{2}-\widetilde{G}_{\mu \nu}^{2} \tilde{\mathbf{l}}_{2}, \\
G_{\mu \nu}=e e_{\mu}^{a} e_{\nu}^{b} l_{a b}, \quad \widetilde{G}_{\mu \nu}=e e_{\mu}^{a} e_{\nu}^{b} \tilde{l}_{a b}, \\
G_{\mu \nu}^{1}=e e_{\mu}^{a} e_{\nu}^{b} l_{a b}^{1}, \quad \widetilde{G}_{\mu \nu}^{1}=e e_{\mu}^{a} e_{\nu}^{b} \tilde{l}_{a b}^{1}, \\
G_{\mu \nu}^{2}=e e_{\mu}^{a} e_{\nu}^{b} l_{a b}^{2}, \quad \widetilde{G}_{\mu \nu}^{2}=e e_{\mu}^{a} e_{\nu}^{b} \tilde{l}_{a b}^{2} .
\end{gathered}
$$


Notice that

$$
\begin{gathered}
\widetilde{G}_{\mu \nu}=\frac{1}{2} \epsilon_{a b c d} \text { e e } e_{\mu}^{a} e_{\nu}^{b} l^{c d}=\frac{e}{2} \epsilon_{\mu \nu c d} l^{c d} \\
=\frac{1}{2} \epsilon_{\mu \nu \rho \sigma} G^{\rho \sigma}=G_{\mu \nu}^{d}, \\
\widetilde{G}_{\mu \nu}^{1}=G_{\mu \nu}^{1 d}, \quad \widetilde{G}_{\mu \nu}^{2}=G_{\mu \nu}^{2 d} .
\end{gathered}
$$

Clearly the two two-forms $G_{\mu \nu}$ and $\widetilde{G}_{\mu \nu}$ can be viewed to represent the restricted metric which are dual to each other. With this (139) has the following compact expression

$$
\begin{gathered}
G_{\mu \nu}\left(\mathcal{D}^{\nu} L^{\rho}-\mathcal{D}^{\rho} L^{\nu}\right)-\widetilde{G}_{\mu \nu}\left(\mathcal{D}^{\nu} \widetilde{L}^{\rho}-\mathcal{D}^{\rho} \widetilde{L}^{\nu}\right) \\
+\mathbf{G}_{\mu \nu} \cdot\left(\hat{D}^{\nu} \mathbf{Z}^{\rho}-\hat{D}^{\rho} \mathbf{Z}^{\nu}\right)=0, \\
\partial_{\mu} G^{\mu \nu}+\mathbf{l} \cdot\left(\mathbf{Z}_{\mu} \times \mathbf{G}^{\mu \nu}\right)=0, \\
\partial_{\mu} \widetilde{G}^{\mu \nu}+\tilde{\mathbf{l}} \cdot\left(\mathbf{Z}_{\mu} \times \mathbf{G}^{\mu \nu}\right)=0, \\
\hat{D}_{\mu} \mathbf{G}^{\mu \nu}+\mathbf{Z}_{\mu} \times\left(G^{\mu \nu} \mathbf{l}-\widetilde{G}^{\mu \nu} \tilde{\mathbf{l}}\right)=0,
\end{gathered}
$$

or equivalently

$$
\begin{gathered}
G_{\mu \nu}\left(\mathcal{D}^{\nu} L^{\rho}-\mathcal{D}^{\rho} L^{\nu}\right)-\widetilde{G}_{\mu \nu}\left(\mathcal{D}^{\nu} \widetilde{L}^{\rho}-\mathcal{D}^{\rho} \widetilde{L}^{\nu}\right) \\
=-G_{\mu \nu}^{i}\left(\mathcal{D}^{\nu} Z_{i}^{\rho}-\mathcal{D}^{\rho} Z_{i}^{\nu}\right)+\widetilde{G}_{\mu \nu}^{i}\left(\mathcal{D}^{\nu} \widetilde{Z}_{i}^{\rho}-\mathcal{D}^{\rho} \widetilde{Z}_{i}^{\nu}\right), \\
\partial_{\mu} G^{\mu \nu}=-\epsilon_{i j}\left(Z_{\mu}^{i} G_{j}^{\mu \nu}-\widetilde{Z}_{\mu}^{i} \widetilde{G}_{j}^{\mu \nu}\right), \\
\partial_{\mu} \widetilde{G}^{\mu \nu}=-\epsilon_{i j}\left(Z_{\mu}^{i} \widetilde{G}_{j}^{\mu \nu}+\widetilde{Z}_{\mu}^{i} G_{j}^{\mu \nu}\right), \\
\partial_{\mu} G_{i}^{\mu \nu}=\epsilon_{i j}\left(L_{\mu} G_{j}^{\mu \nu}-\widetilde{L}_{\mu} \widetilde{G}_{j}^{\mu \nu}-Z_{\mu}^{j} G^{\mu \nu}+\widetilde{Z}_{\mu}^{i} \widetilde{G}^{\mu \nu}\right), \\
\partial_{\mu} \widetilde{G}_{i}^{\mu \nu}=\epsilon_{i j}\left(L_{\mu} \widetilde{G}_{j}^{\mu \nu}+\widetilde{L}_{\mu} G_{j}^{\mu \nu}-Z_{\mu}^{i} \widetilde{G}^{\mu \nu}-\widetilde{Z}_{\mu}^{j} G^{\mu \nu}\right) . \\
\left(i, j=1,2, \quad \epsilon_{12}=-\epsilon_{21}=1\right)
\end{gathered}
$$

This suggests that the valence connection $\mathbf{Z}_{\mu}$ plays the role of the gravitational source of the restricted metric.

In 3-dimensional notation

$$
\begin{gathered}
\hat{\mathbf{g}}_{\mu \nu}=\left(\begin{array}{c}
\hat{m}_{\mu \nu} \\
\hat{e}_{\mu \nu}
\end{array}\right), \quad \mathbf{G}_{\mu \nu}=\left(\begin{array}{c}
\vec{M}_{\mu \nu} \\
\vec{E}_{\mu \nu}
\end{array}\right), \\
\mathbf{g}_{\mu \nu}=\left(\begin{array}{c}
\vec{m}_{\mu \nu} \\
\vec{e}_{\mu \nu}
\end{array}\right)=\left(\begin{array}{c}
\hat{m}_{\mu \nu}+\vec{M}_{\mu \nu} \\
\hat{e}_{\mu \nu}+\vec{E}_{\mu \nu}
\end{array}\right),
\end{gathered}
$$

we have

$$
\begin{gathered}
\hat{m}_{\mu \nu}=G_{\mu \nu} \hat{n}, \quad \hat{e}_{\mu \nu}=\widetilde{G}_{\mu \nu} \hat{n}, \\
\vec{M}_{\mu \nu}=G_{\mu \nu}^{1} \hat{n}_{1}+G_{\mu \nu}^{2} \hat{n}_{2}, \\
\vec{E}_{\mu \nu}=\widetilde{G}_{\mu \nu}^{1} \hat{n}_{1}+\widetilde{G}_{\mu \nu}^{2} \hat{n}_{2},
\end{gathered}
$$

so that the Einstein-Hilbert action (138) acquires the following form

$$
\begin{gathered}
S\left[e_{a}^{\mu}, L_{\mu}, \widetilde{L}_{\mu}, Z_{\mu}^{i}, \widetilde{Z}_{\mu}^{i}\right] \\
=\frac{1}{16 \pi G_{N}} \int\left\{G_{\mu \nu}\left(\mathcal{D}^{\mu} L^{\nu}-\mathcal{D}^{\nu} L^{\mu}\right)\right. \\
-\widetilde{G}_{\mu \nu}\left(\mathcal{D}^{\mu} \widetilde{L}^{\nu}-\mathcal{D}^{\nu} \widetilde{L}^{\mu}\right)+G_{\mu \nu}^{i}\left(\mathcal{D}^{\mu} Z^{\nu i}-\mathcal{D}^{\nu} Z^{\mu i}\right) \\
\left.-\widetilde{G}_{\mu \nu}^{i}\left(\mathcal{D}^{\mu} \widetilde{Z}^{\nu i}-\mathcal{D}^{\nu} \widetilde{Z}^{\mu i}\right)\right\} d^{4} x .
\end{gathered}
$$

From this we can reproduce (144). This completes the $A_{2}$ decomposition (the space-like decomposition) of Einstein's theory.

We can repeat the same procedure with the $B_{2}$ isometry (the light-like isometry) to obtain the desired decomposition of Einstein's equation. With the EinsteinHilbert action

$$
\begin{gathered}
S\left[e_{a}^{\mu}, \Gamma_{\mu}, \widetilde{\Gamma}_{\mu}, \mathbf{Z}_{\mu}\right]=\frac{1}{16 \pi G_{N}} \int\left\{\mathbf{g}_{\mu \nu} \cdot \mathbf{R}_{\mu \nu}\right. \\
\left.+\lambda \mathbf{j}^{2}+\tilde{\lambda}(\mathbf{j} \cdot \tilde{\mathbf{j}})+\lambda_{\mu}\left(\mathbf{k} \cdot \mathbf{Z}^{\mu}\right)+\tilde{\lambda}_{\mu}\left(\tilde{\mathbf{k}} \cdot \mathbf{Z}^{\mu}\right)\right\} d^{4} x, \\
\mathbf{R}_{\mu \nu}=\hat{\mathbf{R}}_{\mu \nu}+\left(\hat{D}_{\mu} \mathbf{Z}_{\nu}-\hat{D}_{\nu} \mathbf{Z}_{\mu}\right)+\mathbf{Z}_{\mu} \times \mathbf{Z}_{\nu} \\
=\left(\mathcal{D}_{\mu} K_{\nu}-\mathcal{D}_{\nu} K_{\mu}\right) \mathbf{j}-\left(\mathcal{D}_{\mu} \widetilde{K}_{\nu}-\mathcal{D}_{\nu} \widetilde{K}_{\mu}\right) \tilde{\mathbf{j}} \\
\quad+\left(\mathcal{D}_{\mu} J_{\nu}-\mathcal{D}_{\nu} J_{\mu}\right) \mathbf{k}-\left(\mathcal{D}_{\mu} \widetilde{J}_{\nu}-\mathcal{D}_{\nu} \widetilde{J}_{\mu}\right) \tilde{\mathbf{k}} \\
\quad+\left(\mathcal{D}_{\mu} L_{\nu}-\mathcal{D}_{\nu} L_{\mu}\right) \mathbf{l}-\left(\mathcal{D}_{\mu} \widetilde{L}_{\nu}-\mathcal{D}_{\nu} \widetilde{L}_{\mu}\right) \tilde{\mathbf{l}}
\end{gathered}
$$

we get following equations of motion

$$
\begin{gathered}
\delta e_{\mu c} ; \quad\left(e e_{\mu}^{a} e_{\nu}^{b}\right)\left(\left(\mathcal{D}^{\nu} K^{\rho}-\mathcal{D}^{\rho} K^{\nu}\right) j_{a b}\right. \\
\left.-\left(\mathcal{D}^{\nu} \widetilde{K}^{\rho}-\mathcal{D}^{\rho} \widetilde{K}^{\nu}\right) \tilde{j}_{a b}+\mathbf{Z}^{\nu \rho} \cdot \mathbf{\Pi}_{a b}\right) e_{\rho c}=0 \\
\delta \Gamma_{\nu} ; \partial_{\mu}\left(e e_{a}^{\mu} e_{b}^{\nu} j^{a b}\right)+\mathbf{j} \cdot\left(\mathbf{Z}_{\mu} \times \mathbf{g}^{\mu \nu}\right)=0 \\
\delta \widetilde{\Gamma}_{\nu} ; \quad \partial_{\mu}\left(e e_{a}^{\mu} e_{b}^{\nu} \tilde{j}^{a b}\right)+\tilde{\mathbf{j}} \cdot\left(\mathbf{Z}_{\mu} \times \mathbf{g}^{\mu \nu}\right)=0 \\
\delta \mathbf{Z}_{\nu} ; \quad \hat{D}_{\mu}\left(e e_{a}^{\mu} e_{b}^{\nu} \mathbf{\Pi}^{a b}\right)+\left(e e_{a}^{\mu} e_{b}^{\nu}\right)\left[\left(\mathbf{Z}_{\mu} \times \mathbf{k}\right) j^{a b}\right. \\
\left.-\left(\mathbf{Z}_{\mu} \times \tilde{\mathbf{k}}\right) \tilde{j}^{a b}\right]=0,
\end{gathered}
$$

where now

$$
\begin{gathered}
\boldsymbol{\Pi}_{a b}=k_{a b} \mathbf{j}-\tilde{k}_{a b} \tilde{\mathbf{j}}+l_{a b} \mathbf{l}-\tilde{l}_{a b} \tilde{\mathbf{l}}=\mathbf{I}_{a b}-\boldsymbol{\Sigma}_{a b}, \\
\boldsymbol{\Sigma}_{a b}=j_{a b} \mathbf{k}-\tilde{j}_{a b} \tilde{\mathbf{k}} .
\end{gathered}
$$

Notice that here $\boldsymbol{\Pi}_{a b}$ and $\boldsymbol{\Sigma}_{a b}$ do not make projection operators, because

$$
\boldsymbol{\Pi}_{a b} \cdot \boldsymbol{\Sigma}^{c d}=k_{a b} j^{c d}-\tilde{k}_{a b} \tilde{j}^{c d} \neq 0 .
$$

Now, again we can combine the last three equations of (149) into a single equation with the help of $\hat{D}_{\mu} \mathbf{j}=\hat{D}_{\mu} \tilde{\mathbf{j}}=$ 0 ,

$$
D_{\mu} \mathbf{g}^{\mu \nu}=0 .
$$

This confirms that (149) is equivalent to (133), which tells that (148) describes the Einstein's gravity.

Now, with

$$
\begin{gathered}
\mathbf{g}_{\mu \nu}=\hat{\mathbf{g}}_{\mu \nu}+\mathbf{G}_{\mu \nu}, \\
\hat{\mathbf{g}}_{\mu \nu}=e e_{\mu}^{a} e_{\nu}^{b} \boldsymbol{\Sigma}^{a b}=\mathcal{J}_{\mu \nu} \mathbf{k}-\widetilde{\mathcal{J}}_{\mu \nu} \tilde{\mathbf{k}}, \\
\mathbf{G}_{\mu \nu}=e e_{\mu}^{a} e_{\nu}^{b} \mathbf{\Pi}^{a b} \\
=\mathcal{K}_{\mu \nu} \mathbf{j}-\widetilde{\mathcal{K}}_{\mu \nu} \tilde{\mathbf{j}}+\mathcal{L}_{\mu \nu} \mathbf{l}-\widetilde{\mathcal{L}}_{\mu \nu} \tilde{\mathbf{l}}, \\
\mathcal{J}_{\mu \nu}=e e_{\mu}^{a} e_{\nu}^{b} j_{a b}, \quad \widetilde{\mathcal{J}}_{\mu \nu}=e e_{\mu}^{a} e_{\nu}^{b} \tilde{j}_{a b}, \\
\mathcal{K}_{\mu \nu}=e e_{\mu}^{a} e_{\nu}^{b} k_{a b}, \quad \widetilde{\mathcal{K}}_{\mu \nu}=e e_{\mu}^{a} e_{\nu}^{b} \tilde{k}_{a b}, \\
\mathcal{L}_{\mu \nu}=e e_{\mu}^{a} e_{\nu}^{b} l_{a b}, \quad \widetilde{\mathcal{L}}_{\mu \nu}=e e_{\mu}^{a} e_{\nu}^{b} \tilde{l}_{a b},
\end{gathered}
$$


the equation (149) is written as

$$
\begin{gathered}
\mathcal{J}_{\mu \nu}\left(\mathcal{D}^{\nu} K^{\rho}-\mathcal{D}^{\rho} K^{\nu}\right)-\widetilde{\mathcal{J}}_{\mu \nu}\left(\mathcal{D}^{\nu} \widetilde{K}^{\rho}-\mathcal{D}^{\rho} \widetilde{K}^{\nu}\right) \\
+\mathbf{G}_{\mu \nu} \cdot \mathbf{Z}^{\nu \rho}=0 \\
\partial_{\mu} \mathcal{J}^{\mu \nu}+\mathbf{j} \cdot\left(\mathbf{Z}_{\mu} \times \mathbf{G}^{\mu \nu}\right)=0 \\
\partial_{\mu} \widetilde{\mathcal{J}}^{\mu \nu}+\tilde{\mathbf{j}} \cdot\left(\mathbf{Z}_{\mu} \times \mathbf{G}^{\mu \nu}\right)=0 \\
\hat{D}_{\mu} \mathbf{G}^{\mu \nu}+\mathbf{Z}_{\mu} \times\left(\mathcal{J}_{\mu \nu} \mathbf{k}-\widetilde{\mathcal{J}}_{\mu \nu} \tilde{\mathbf{k}}\right)=0
\end{gathered}
$$

or equivalently

$$
\begin{gathered}
\mathcal{J}_{\mu \nu}\left(\mathcal{D}^{\nu} K^{\rho}-\mathcal{D}^{\rho} K^{\nu}\right)-\widetilde{\mathcal{J}}_{\mu \nu}\left(\mathcal{D}^{\nu} \widetilde{K}^{\rho}-\mathcal{D}^{\rho} \widetilde{K}^{\nu}\right) \\
=-\mathcal{K}_{\mu \nu}\left(\mathcal{D}^{\nu} J^{\rho}-\mathcal{D}^{\rho} J^{\nu}\right)+\widetilde{\mathcal{K}}_{\mu \nu}\left(\mathcal{D}^{\nu} \widetilde{J}^{\rho}-\mathcal{D}^{\rho} \widetilde{J}^{\nu}\right) \\
-\mathcal{L}_{\mu \nu}\left(\mathcal{D}^{\nu} L^{\rho}-\mathcal{D}^{\rho} L^{\nu}\right)+\widetilde{\mathcal{L}}_{\mu \nu}\left(\mathcal{D}^{\nu} \widetilde{L}^{\rho}-\mathcal{D}^{\rho} \widetilde{L}^{\nu}\right), \\
\partial_{\mu} \mathcal{J}^{\mu \nu}=L_{\mu} \widetilde{\mathcal{J}}^{\mu \nu}+\widetilde{L}_{\mu} \mathcal{J}^{\mu \nu}-J_{\mu} \widetilde{\mathcal{L}}^{\mu \nu}-\widetilde{J}_{\mu} \mathcal{L}^{\mu \nu}, \\
\partial_{\mu} \widetilde{\mathcal{J}}^{\mu \nu}=-L_{\mu} \mathcal{J}^{\mu \nu}+\widetilde{L}_{\mu} \widetilde{\mathcal{J}}^{\mu \nu}+J_{\mu} \mathcal{L}^{\mu \nu}-\widetilde{J}_{\mu} \widetilde{\mathcal{L}}^{\mu \nu} \\
\partial_{\mu} \mathcal{K}^{\mu \nu}=-L_{\mu} \widetilde{\mathcal{K}}^{\mu \nu}-\widetilde{L}_{\mu} \mathcal{K}^{\mu \nu}-K_{\mu} \mathcal{L}^{\mu \nu}+\widetilde{K}_{\mu} \widetilde{\mathcal{L}}^{\mu \nu}, \\
\partial_{\mu} \widetilde{\mathcal{K}}^{\mu \nu}=L_{\mu} \mathcal{K}^{\mu \nu}-\widetilde{L}_{\mu} \widetilde{\mathcal{K}}^{\mu \nu}+K_{\mu} \widetilde{\mathcal{L}}^{\mu \nu}+\widetilde{K}_{\mu} \mathcal{L}^{\mu \nu}, \\
\partial_{\mu} \mathcal{L}^{\mu \nu}=K_{\mu} \mathcal{J}^{\mu \nu}-\widetilde{K}_{\mu} \widetilde{\mathcal{J}}^{\mu \nu}+J_{\mu} \widetilde{\mathcal{K}}^{\mu \nu}+\widetilde{J}_{\mu} \mathcal{K}^{\mu \nu}, \\
\partial_{\mu} \widetilde{\mathcal{L}}^{\mu \nu}=-K_{\mu} \widetilde{\mathcal{J}}^{\mu \nu}-\widetilde{K}_{\mu} \mathcal{J}^{\mu \nu} \\
-J_{\mu} \mathcal{K}^{\mu \nu}+\widetilde{J}_{\mu} \widetilde{\mathcal{K}}^{\mu \nu} .
\end{gathered}
$$

Remember that $\mathcal{J}_{\mu \nu}, \mathcal{K}_{\mu \nu}, \mathcal{L}_{\mu \nu}$ and $\widetilde{\mathcal{J}}_{\mu \nu}, \widetilde{\mathcal{K}}_{\mu \nu}, \widetilde{\mathcal{L}}_{\mu \nu}$ are dual to each other. Here again the valence connection becomes the gravitational source of the restricted metric.

In 3-dimensional notation we have

$$
\begin{gathered}
\mathbf{g}_{\mu \nu}=\left(\begin{array}{c}
\vec{m}_{\mu \nu} \\
\vec{e}_{\mu \nu}
\end{array}\right)=\left(\begin{array}{c}
\hat{m}_{\mu \nu}+\vec{M}_{\mu \nu} \\
\hat{e}_{\mu \nu}+\vec{E}_{\mu \nu}
\end{array}\right), \\
\hat{m}_{\mu \nu}=\frac{e^{-\lambda}}{\sqrt{2}}\left(\mathcal{J}_{\mu \nu} \hat{n}_{1}+\widetilde{\mathcal{J}}_{\mu \nu} \hat{n}_{2}\right), \\
\hat{e}_{\mu \nu}=\frac{e^{-\lambda}}{\sqrt{2}}\left(\widetilde{\mathcal{J}}_{\mu \nu} \hat{n}_{1}-\mathcal{J}_{\mu \nu} \hat{n}_{2}\right), \\
\hat{m}_{\mu \nu}=\vec{n}_{3} \times \hat{e}_{\mu \nu}, \quad \hat{e}_{\mu \nu}=-\vec{n}_{3} \times \hat{m}_{\mu \nu}, \\
\vec{M}_{\mu \nu}=\frac{e^{\lambda}}{\sqrt{2}}\left(\mathcal{K}_{\mu \nu} \hat{n}_{1}+\widetilde{\mathcal{K}}_{\mu \nu} \hat{n}_{2}\right)+\mathcal{L}_{\mu \nu} \hat{n}_{3}, \\
\vec{E}_{\mu \nu}=\frac{e^{\lambda}}{\sqrt{2}}\left(-\widetilde{\mathcal{K}}_{\mu \nu} \hat{n}_{1}+\mathcal{K}_{\mu \nu} \hat{n}_{2}\right)+\widetilde{\mathcal{L}}_{\mu \nu} \hat{n}_{3},
\end{gathered}
$$

so that the Einstein-Hilbert action (148) is expressed as

$$
\begin{gathered}
S\left[e_{a}^{\mu}, K_{\mu}, \widetilde{K}_{\mu}, J_{\mu}, \widetilde{J}_{\mu}, L_{\mu}, \widetilde{L}_{\mu}\right] \\
=\frac{1}{16 \pi G_{N}} \int\left\{\mathcal{J}_{\mu \nu}\left(\mathcal{D}^{\mu} K^{\nu}-\mathcal{D}^{\nu} K^{\mu}\right)\right. \\
-\widetilde{\mathcal{J}}_{\mu \nu}\left(\mathcal{D}^{\mu} \widetilde{K}^{\nu}-\mathcal{D}^{\nu} \widetilde{K}^{\mu}\right)+\mathcal{K}_{\mu \nu}\left(\mathcal{D}^{\mu} J^{\nu}-\mathcal{D}^{\nu} J^{\mu}\right) \\
-\widetilde{\mathcal{K}}_{\mu \nu}\left(\mathcal{D}^{\mu} \widetilde{J}^{\nu}-\mathcal{D}^{\nu} \widetilde{J}^{\mu}\right)+\mathcal{L}_{\mu \nu}\left(\mathcal{D}^{\mu} L^{\nu}-\mathcal{D}^{\nu} L^{\mu}\right) \\
\left.-\widetilde{\mathcal{L}}_{\mu \nu}\left(\mathcal{D}^{\mu} \widetilde{L}^{\nu}-\mathcal{D}^{\nu} \widetilde{L}^{\mu}\right)\right\} d^{4} x .
\end{gathered}
$$

From this we can reproduce (154). This completes the $B_{2}$ decomposition (the light-like decomposition) of Einstein's theory.
The above decompositions of Einstein's theory are interesting in their own right. They allow us a new interpretation of Einstein's theory which sheds a new light on gravitation. More importantly they have far reaching consequences, as we will see [29].

\section{DISCUSSIONS}

In this paper we have discussed the Abelian decomposition of Einstein's theory. Imposing proper magnetic isometries to the gravitational connection, we have shown how to decompose the gravitational connection and the curvature tensor into the restricted part of the maximal Abelian subgroup $H$ of Lorentz group $G$ and the valence part of $G / H$ component which plays the role of the Lorentz covariant gravitational source of the restricted connection, without compromising the general invariance. This tells that the Einstein's theory can be viewed as a theory of the restricted gravity made of the restricted connection in which the valence connection plays the role of the gravitational source of the restricted gravity. We show that there are two different Abelian decompositions of Einstein's theory, the space-like $A_{2}$ decomposition and the light-like $B_{2}$ decomposition, because Lorentz group has two maximal Abelian subgroups.

A common difficulty in non-Abelian gauge theory and gravitation is the highly non-linear self interaction. In gauge theory one can simplify this non-linear interaction by separating the gauge covariant valence part from the Abelian part of the potential. This simplification has played a crucial role to establish the Abelian dominance in non-Abelian gauge theory. Here we have shown that we can simplify the gravitational interaction exactly the same way, treating Einstein's theory as a gauge theory of Lorentz group.

An important ingredient of the decomposition is the concept of Lorentz covariant four-index metric tensor $\mathbf{g}_{\mu \nu}$ which replaces the role of the two-index space-time metric $g_{\mu \nu}$. We have shown that the metric-compatibility condition of the connection $\nabla_{\alpha} g_{\mu \nu}=0$ is replaced by the gauge covariant condition $D_{\mu} \mathbf{g}^{\mu \nu}=0$.

From theoretical point of view, the above decomposition of gravitation differs from the Abelian decomposition of non-Abelian gauge theory in one important respect. In gauge theory the fundamental ingredient is the gauge potential, and the decomposition of the potential provides a complete decomposition of the theory. On the other hand in gravitation the fundamental field is assumed to be the metric, not the potential (the connection), but our decomposition is based on the connection. So one might wonder whether one can have an Abelian decomposition of Einstein's theory based on the metric. Of course our decomposition allows us to have the the decomposition of the metric, but only indirectly through the equation of motion, as we have seen. It would be very interesting to 
see if one can actually decompose the metric explicitly, and decompose Einstein's theory in terms of the metric.

This touches a subtle but very important issue on the essence of gravitation. The issue here is what describes gravity. In Einstein's theory gravity is described by the metric alone. But, as Cartan has pointed out long time ago, torsion could also be an important part of gravity [26]. And indeed in the gauge formalism of Einstein's theory the connection (the gauge potential of Lorentz group) naturally includes torsion [10, 11]. This tells that our Abelian decomposition is a decomposition of a generalized Einstein's theory which includes torsion. This is very important, because torsion could generate new topological structures which are absent in Einstein's gravity.

Clearly the above decomposition of (generalized) Einstein's theory has many interesting implications. First of all, this tells that one can actually construct a restricted theory of gravitation made of the restricted part of connection alone which has the full Lorentz invariance, or equivalently the full general invariance, by excluding the Lorentz covariant valence part of connection from the theory. In other words, one can have a theory of gravitation which actually is much simpler than Einstein's theory, which nevertheless has the full general invariance and thus retains all topological characteristics of Einstein's theory. This is very important, because this tells that the restricted gravity describes the core dynamics of Einstein's theory. This point can play a crucial role for us to understand the quantum gravity.

Furthermore, the decomposition makes the topology of generalized Einstein's theory more transparent as well as interesting. Indeed with the Abelian decomposition we can study the topological structures of the theory more easily, because the topological characteristics are imprinted in the magnetic symmetry. For example, the $A_{2}$ decomposition makes it clear that the topology of Einstein's theory is closely related to the topology of $S U(2)$ gauge theory. This is natural, because $S U(2)$ forms a subgroup of Lorentz group. This strongly implies that gravity may allow the gravito-magnetic monopole which has the monopole topology $\pi_{2}\left(S^{2}\right)$ [27, 28].

Perhaps more importantly, this strongly implies that classical space-time in generalized Einstein's theory may have the multiple vacua similar to what we find in $S U(2)$ gauge theory. This turns out to be true. In fact with a proper magnetic isometry we can construct all possible vacuum space-times, and show that they are classified by exactly the same multiple vacua that we have in $S U(2)$ gauge theory which can be classified by the knot topology $\pi_{3}\left(S^{3}\right)=\pi_{3}\left(S^{2}\right)[29]$. This has a far reaching consequence. Just as in $S U(2)$ gauge theory, the multiple vacua in gravity can be unstable against quantum fluctuation. And there is a real possibility that gravitational interaction may allow the gravito-instantons which can connect topologically distinct vacua and thus allow the vacuum tunnelling. Clearly this will have an important implication in quantum gravity. Of course the "gravitational istantons" which have finite action in Euclidian space-time have been discussed before, because it was hoped that these Euclidian configurations could play an important role in quantum gravity [30, 31]. But so far it has not been known exactly what role they play in quantum gravity. In any case they have not been associated with the above multiple vacuum space-time.

The above discussions raise more questions need to be answered. A first question is the existence of a real gravito-instanton which can actually make the quantum tunnelling between topologically different vacuum space-times. It would be intersting to find such gravitoinstanton (if exist), and see whether any of the known finite action Euclidian configurations can make the desired quantum tunnelling. Another question is the role (if any) of torsion in this multiple vacuua and vacuum tunneling. Again it would be important to know whether Einstein's theory in the absence of torsion can admit multiple vacuua and/or vacuum tunneling. If this is so, it would be very interesting to construct the multiple vacuua and gravito-instanton in terms of metric. The details of the subject and related issues will be discussed separately in an accompanying paper [32].

\section{ACKNOWLEDGEMENT}

The work is supported in part by the BSR Program (Grant KRF-2007-314-C00055) of Korea Research Foundation and in part by the ABRL Program (Grant R14-2003-012-01002-0) and the International Cooperation Program of Korea Science and Engineering Foundation.
[1] T. Kaluza, Sitzber. Preuss. Akad. Wiss. 966 (1921); O. Klein, Z. Physik 37, 895 (1926).

[2] P. Jordan, Z. Phys. 157, 112 (1959); Y. Thirry, C. R. Acad. Sci. Paris 226, 216 (1948); R. Kerner, Ann. Inst. H. Poincare 9, 143 (1968); A. Trautman, Rep. Math. Phys. 1, 29 (1970).

[3] Y. M. Cho, J. Math. Phys. 16, 2029 (1975); Y. M. Cho and Pongsoo Zhang, Phys. Rev. D12, 3789 (1975).

[4] Y. M. Cho and P. G. O. Freund, Phys. Rev. D12, 1711
(1975).

[5] Y. M. Cho, Phys. Lett. B186, 38 (1987); J. Math. Phys. 30, 1570 (1989).

[6] T. W. B. Kibble, J. Math. Phys. 2, 212 (1961); D. Sciama, Rev. Mod. Phys. 36, 463 (1964).

[7] R. Utiyama, Phys. Rev. 101, 1597 (1956); R. Utiyama and T. Fukuyama, Prog. Theor. Phys. 45, 612 (1971).

[8] M. Carmelli, J. Math. Phys. 11, 2728 (1970).

[9] Y. M. Cho, Phys. Rev. D14, 2521 (1976); See also 100 
Years of Gravity and Accelerated Frames - The Deepest Insights of Einstein and Yang-Mills, edited by J. P. Hsu, World Scientific, 2006.

[10] Y. M. Cho, Phys. Rev. D14, 3335 (1976).

[11] See also, F. Hehl, P. Heyde, G. Kerlick, and J. Nester, Rev. Mod. Phys. 48, 393 (1976).

[12] Y. M. Cho, Phys. Rev. D21, 1080 (1980); Phys. Rev D62, 074009 (2000).

[13] Y. M. Cho, Phys. Rev. Lett. 46, 302 (1981); Phys. Rev. D23, 2415 (1981); W. S. Bae, Y. M. Cho, and S. W. Kimm, Phys. Rev. D65, 025005 (2002).

[14] L. Faddeev and A. Niemi, Phys. Rev. Lett. 82, 1624 (1999); Phys. Lett. B449, 214 (1999); S. Shabanov, Phys. Lett. B458, 322 (1999); B463, 263 (1999); H. Gies, Phys. Rev. D63, 125023 (2001); R. Zucchini, Int. J. Geom. Meth. Mod. Phys. 1, 813 (2004).

[15] Y. M. Cho, H. W. Lee, and D. G. Pak, Phys. Lett. B 525, 347 (2002); Y. M. Cho and D. G. Pak, Phys. Rev. D65, 074027 (2002).

[16] Y. M. Cho, D. G. Pak, and M. Walker, JHEP 05, 073 (2004); Y. M. Cho and M. L. Walker, Mod. Phys. Lett. A19, 2707 (2004).

[17] K. Kondo, Phys. Lett. B600, 287 (2004); K. Kondo, T. Murakami, and T. Shinohara, Euro Phys. J. C42, 475 (2005); S. Kato et. al., Phys. Lett. B632, 326 (2006).

[18] Y. M. Cho, Phys. Rev. Lett. 68, 3133 (1992).

[19] M. Duff, B. Nilsson, and C. Pope, Phys. Rep. 130, 1 (1986).

[20] This point has also been emphasized recently by Penrose.
See R. Penrose, The Road to Reality : A Complete Guide to The Laws of The Universe, A.A. Knopf, 2005.

[21] Y. M. Cho, Phys. Rev. Lett. 44, 1115 (1980); Phys. Lett. B115, 125 (1982).

[22] A. Belavin, A. Polyakov, A. Schwartz, and Y. Tyupkin, Phys. Lett. B59, 85 (1975); Y. M. Cho, Phys. Lett. B81, 25 (1979).

[23] P. van Baal and A. Wipf, Phys. Lett. B515, 181 (2001).

[24] Y. M. Cho, Phys. Lett. B644, 208 (2006).

[25] Y. M. Cho, J. Math. Phys. 20, 2605 (1979).

[26] E. Cartan, C. R. Acad. Sci. Paris 174, 593 (1922); Ann. Ecole Normale 41, 1 (1924).

[27] A. Zee, Phys. Rev. Lett. 55, 2379 (1985); Y. M. Cho, in Proceedings of XXV International Conference on High Energy Physics, edited by Y. Yamaguchi and K. K. Phua, World Scientific (Singapore) 1991.

[28] E. Newman, L. Tamburino, and Unti, J. Math. Phys. 4, 915 (1963); C. Misner, ibid., 924 (1963); J. Dowker, Gen. Rel. Grav. 5, 603 (1974); S. Ramaswamy and A. Sen, J. Math. Phys. 22, 2612 (1981).

[29] Y. M. Cho, hep-th/0703016.

[30] S. Hawking, Phys. Lett. A60, 81 (1977); T. Eguchi and A. Hanson, Phys. Lett. B74, 249 (1978); G. Gibbons and S. Hawking, Phys. Lett. B78, 430 (1978).

[31] See also, T. Eguchi, P. Gilkey, and A. Hanson, Phys. Rep. 66, 213 (1980).

[32] Y. M. Cho, Prog. Theoretical Phys. (Japan J. Phys. Soc.) in press; Y. M. Cho and S. W. Kim, to be published. 